\title{
Autoantibodies against thrombospondin type 1 domain-containing 7A induce membranous nephropathy
}

\author{
Nicola M. Tomas, ${ }^{1}$ Elion Hoxha, ${ }^{1}$ Anna T. Reinicke, ${ }^{1}$ Lars Fester, ${ }^{2}$ Udo Helmchen, ${ }^{3}$ Jens Gerth, ${ }^{4}$ Friederike Bachmann, ${ }^{5}$ \\ Klemens Budde, ${ }^{5}$ Friedrich Koch-Nolte, ${ }^{6}$ Gunther Zahner, ${ }^{1}$ Gabriele Rune, ${ }^{2}$ Gerard Lambeau, ${ }^{7}$ \\ Catherine Meyer-Schwesinger, ${ }^{1}$ and Rolf A.K. Stahl ${ }^{1}$
}

${ }^{1}$ III Medizinische Klinik, ${ }^{2}$ Institut für Neuroanatomie, and ${ }^{3}$ Nierenregister, Universitätsklinikum Hamburg-Eppendorf, Hamburg, Cermany. ${ }^{4}$ Klinik für Innere Medizin II, Heinrich-Braun-Klinikum Zwickau,

Zwickau, Germany. ${ }^{5}$ Medizinische Klinik mit Schwerpunkt Nephrologie, Charité - Universitätsmedizin Berlin Campus Mitte, Berlin, Germany. ${ }^{6}$ Institut für Immunologie, Universitätsklinikum Hamburg-

Eppendorf, Hamburg, Germany. ${ }^{7}$ Institut de Pharmacologie Moléculaire et Cellulaire, UMR7275, Centre National de la Recherche Scientifique and Université de Nice Sophia Antipolis, Valbonne, France.

\begin{abstract}
Membranous nephropathy (MN) is the most common cause of nephrotic syndrome in adults, and one-third of patients develop end-stage renal disease (ESRD). Circulating autoantibodies against the podocyte surface antigens phospholipase $A_{2}$ receptor $1\left(P L A_{2} R 1\right)$ and the recently identified thrombospondin type 1 domain-containing 7A (THSD7A) are assumed to cause the disease in the majority of patients. The pathogenicity of these antibodies, however, has not been directly proven. Here, we have reported the analysis and characterization of a male patient with THSD7A-associated MN who progressed to ESRD and subsequently underwent renal transplantation. MN rapidly recurred after transplantation. Enhanced staining for THSD7A was observed in the kidney allograft, and detectable anti-THSD7A antibodies were present in the serum before and after transplantation, suggesting that these antibodies induced a recurrence of $M N$ in the renal transplant. In contrast to PLA $R 1$, THSD7A was expressed on both human and murine podocytes, enabling the evaluation of whether anti-THSD7A antibodies cause MN in mice. We demonstrated that human anti-THSD7A antibodies specifically bind to murine THSD7A on podocyte foot processes, induce proteinuria, and initiate a histopathological pattern that is typical of MN. Furthermore, anti-THSD7A antibodies induced marked cytoskeletal rearrangement in primary murine glomerular epithelial cells as well as in human embryonic kidney 293 cells. Our findings support a causative role of anti-THSD7A antibodies in the development of MN.
\end{abstract}

\section{Introduction}

Membranous nephropathy (MN) is an autoimmune disease that is histologically characterized by thickening of the glomerular basement membrane (GBM), granular staining for IgG, positivity for components of the complement system, and the presence of electron-dense deposits in the subepithelial space and within the GBM. Clinically, most patients present with high levels of proteinuria that usually exceed 3.5 grams per day, in conjunction with a nephrotic syndrome. The pathophysiology of MN has mainly been studied in the rat model of Heymann nephritis $(1,2)$. In passive Heymann nephritis, the transfer of sheep antibodies against the podocyte membrane protein megalin results in subepithelial immune complex formation $(3,4)$, activation of the complement system (5), and development of proteinuria. The concept that human $\mathrm{MN}$ is an antibody-mediated autoimmune disease has been supported by the discoveries of neutral endopeptidase (NEP) (6), phospholipase $\mathrm{A}_{2}$ receptor 1 ( $\left.\mathrm{PLA}_{2} \mathrm{R} 1\right)$ (7), and thrombospondin type 1 domain-containing 7A (THSD7A) (8) as podocyte membrane proteins serving as antigens in this disease. The current

Authorship note: N.M. Tomas and E. Hoxha, as well as C. Meyer-Schwesinger and R.A.K. Stahl, contributed equally to this work.

Conflict of interest: The authors have declared that no conflict of interest exists. Submitted: October 28, 2015; Accepted: March 31, 2016.

Reference information: / Clin Invest. 2016;126(7):2519-2532. doi:10.1172/JCI85265. view is that PLA 21 and THSD7A are targets for a malfunctioning immune system in $70 \%$ and $5 \%$ of adult cases, respectively, and that NEP is important in a small number of neonates with MN caused by alloimmunization due to the vertical transfer of antibodies from a genetically NEP-deficient mother (9). The findings that antibodies against $\mathrm{PLA}_{2} \mathrm{R} 1$ are present in patients with a recurrence of $\mathrm{MN}$ after renal transplantation $(10,11)$ and that anti$\mathrm{PLA}_{2} \mathrm{R} 1$ antibody levels are associated with disease remission (12, 13) and progression (13-15) strongly support a pathogenic role of these antibodies in the development of MN. However, attempts to provide final proof that anti-PLA ${ }_{2} \mathrm{R} 1$ antibodies indeed cause $\mathrm{MN}$ have been hampered by the fact that PLA $\mathrm{R}_{2} 1$ is not expressed on rodent podocytes. Given the recent observations that THSD7A, in contrast to $\mathrm{PLA}_{2} \mathrm{R} 1$, is expressed on both human and murine podocytes $(16,17)$, we investigated whether anti-THSD7A antibodies can cause $\mathrm{MN}$ in mice.

\section{Results}

Serum anti-THSD7A antibody positivity and recurrence of $M N$ after renal transplantation. In 2004, a 57-year-old male was diagnosed with MN. An extensive clinical workup including medical history, prior medication, diagnostic tests for infections (hepatitis B and C, HIV, tuberculosis, treponema) and autoimmune diseases (lupus, rheumatoid arthritis, antineutrophil cytoplasmic antibody-associated [ANCA-associated] vasculitis, anti-GBM disease, sar- 
A Native kidney 2005

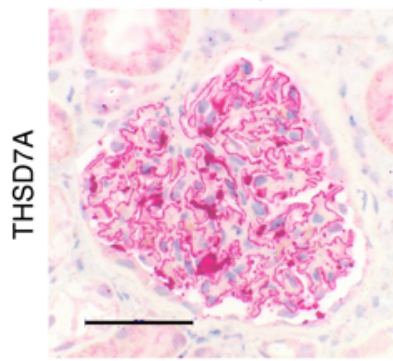

Renal transplant 2014
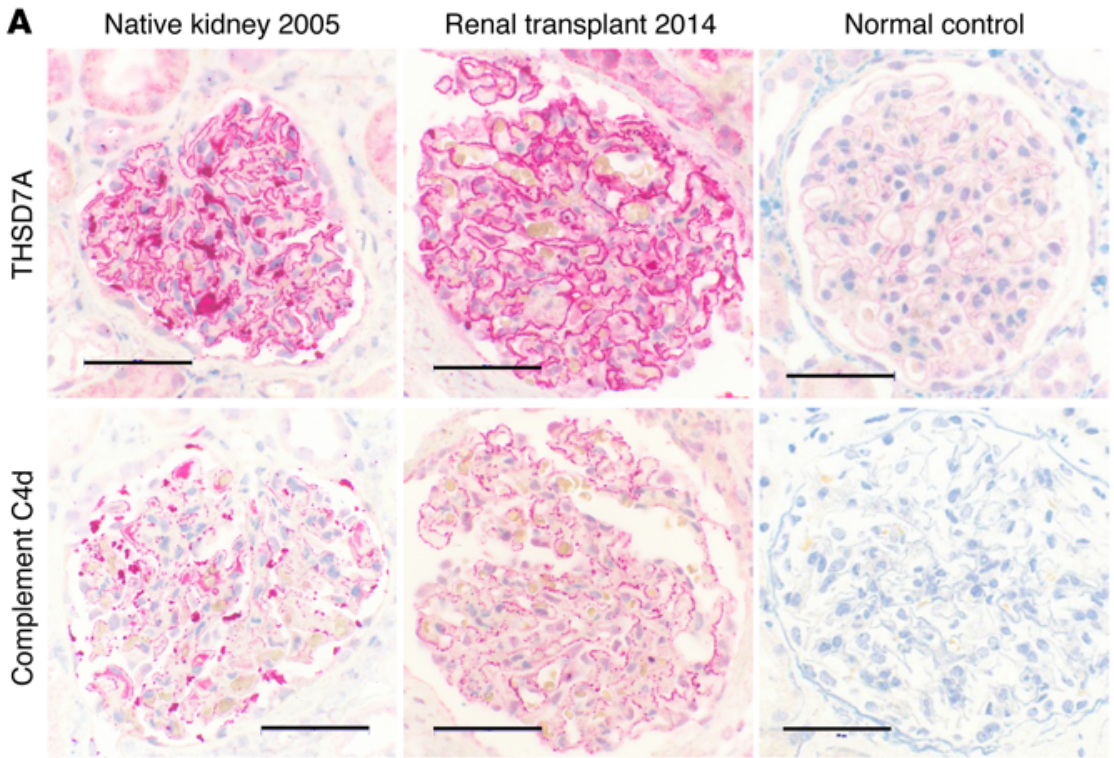

B

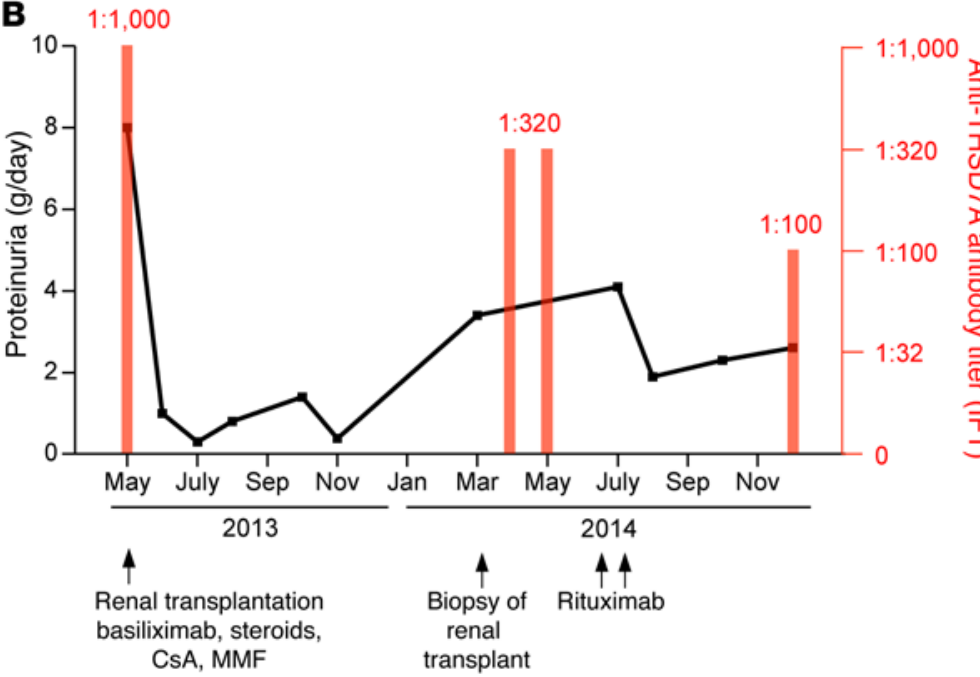

Figure 1. Serum anti-THSD7A antibody positivity and recurrence of $\mathrm{MN}$ after renal transplantation. (A) Immunohistochemical staining for THSD7A and complement C4d in biopsies from a patient with circulating anti-THSD7A antibodies before and after renal transplantation. Scale bars: $50 \mu \mathrm{m}$. (B) Clinical course of the same patient showing 24-hour proteinuria and serum anti-THSD7A antibody titers when measured with an IFT. Serum antiTHSD7A antibody levels were high at the time of transplantation and at the time of MN recurrence in the renal allograft. CsA, cyclosporine A; MMF, mycophenolate mofetil.

however, the patient's proteinuria increased to 3.4 grams per day, and a subsequent renal transplant biopsy showed a recurrence of $\mathrm{MN}$, with normal staining for PLA ${ }_{2} \mathrm{R} 1$, enhanced staining for THSD7A, and positivity for complement C4d (Figure 1A and Supplemental Table 1). At that time, no donor-specific antibodies were detectable, and no evidence of a concomitant antibody-mediated rejection or another condition that might have led to a secondary MN was present. The patient's serum creatinine levels increased shortly thereafter (Supplemental Figure 1), and anti-THSD7A antibodies were detectable, with a titer of 1:320 (Figure 1B). As proteinuria continued to rise, the patient received 2 doses of rituximab in addition to the continuous immunosuppression. Subsequently, the proteinuria decreased, but persisted at approximately 2.5 grams per day. Anti-THSD7A antibody levels also diminished, but remained positive at a level of 1:100 (Figure 1B). Taken together, this clinical case suggests a causative role of persisting circulating anti-THSD7A antibodies in

coidosis), chest x-ray, chest CT, gastroscopy, colonoscopy, and bronchoscopy did not reveal any secondary cause of disease. In addition to supportive treatment with angiotensin-converting enzyme (ACE) inhibitors, statins, diuretics, and anticoagulants, the patient was treated with glucocorticoids and cyclosporine A. The patient's renal function deteriorated, and a second renal biopsy was performed in 2005, again showing MN, with severe tubulointerstitial scarring, normal staining for $\mathrm{PLA}_{2} \mathrm{R} 1$, enhanced staining for THSD7A (stained retrospectively on archival tissue), and positivity for complements C3 and C4d (Figure 1A and Supplemental Table 1; supplemental material available online with this article; doi:10.1172/JCI85265DS1). In the same year, the patient progressed to end-stage renal disease (ESRD), and dialysis was started. In May 2013, the patient received a kidney transplant from a deceased donor. At the time of transplantation, serum anti-THSD7A antibody levels were positive, with a titer of 1:1,000 (Figure 1B). After transplantation, the patient received standard immunosuppressive treatment, proteinuria decreased, and renal function normalized (Supplemental Figure 1). In March 2014, the recurrence of $\mathrm{MN}$ in the transplanted kidney.

Human anti-THSD7A autoantibodies bind to mouse glomerular THSD7A. Serum samples from 2 patients with anti-THSD7A antibody-positive MN were taken for further studies (Supplemental Figure 2 and Supplemental Table 2). Our experiments demonstrated the ability of human anti-THSD7A antibodies to bind to the mouse antigen on podocyte foot processes in vitro and in vivo. Precisely, anti-THSD7A antibody-containing sera immunoprecipitated THSD7A from mouse glomeruli (Figure 2A) and bound linearly along the glomerular capillary wall in colocalization with nephrin in indirect immunofluorescence analyses of naive murine kidneys (Figure 2B). In vivo analyses further demonstrated binding of huIgG4 (huIgG4), the predominant anti-THSD7A IgG subclass in the patient's serum, exclusively along the glomerular filtration barrier in colocalization with nephrin 2 hours after i.v. injection of anti-THSD7A antibody-containing serum into mice (Figure 2C).

Human anti-THSD7A autoantibodies induce the histological features of MN in mice. On the basis of the above-described clinical data, our observation that rodents express THSD7A on glo- 
A IP: sera

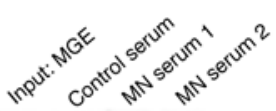

Blot: anti-THSD7A - - $-4250 \mathrm{kDa}$

Blot: anti-human IgG

$450 \mathrm{kDa}$

B

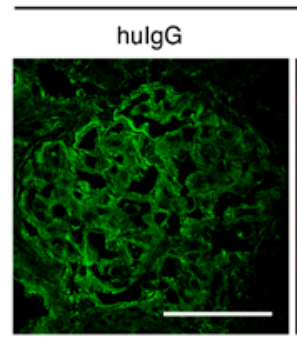

Anti-THSD7A positive

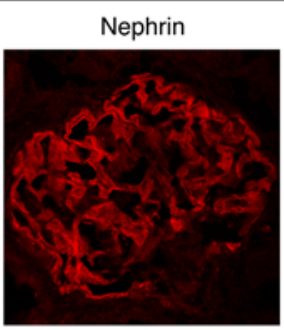

Anti-THSD7A positive

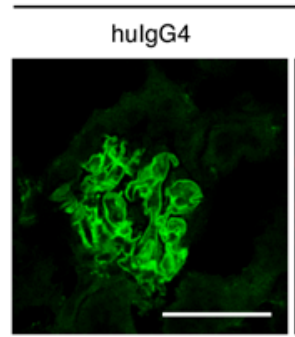

Nephrin

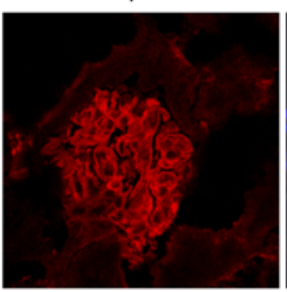

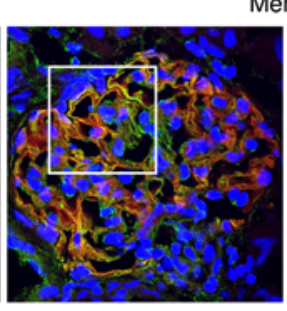

Merge
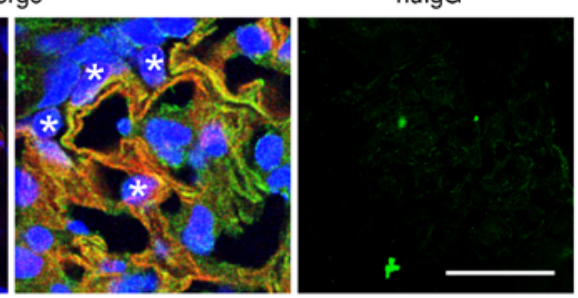

Control serum

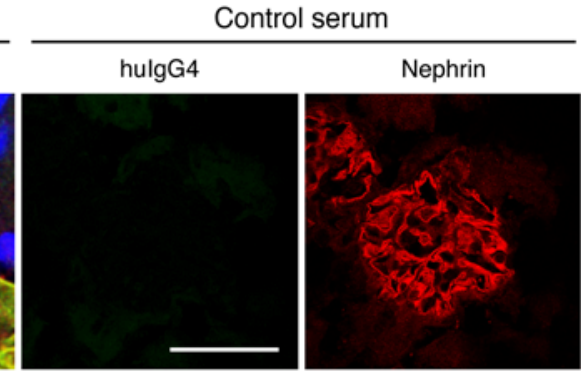

Figure 2. Human anti-THSD7A antibodies bind to mouse THSD7A in vitro and in vivo. (A) Sera from 2 patients with anti-THSD7A antibody-positive MN, but not serum from a healthy individual, immunoprecipitated THSD7A from MCEs. (B) Indirect immunofluorescence analysis using serum from a patient with THSD7A-associated MN or control serum in frozen sections of mouse kidneys. Original magnification, $\times 1,000,4 \times$ zoom (enlargement of the boxed area). (C) Immunofluorescence staining for hulgG4 and nephrin 2 hours after i.v. injection of $100 \mu \mathrm{l}$ serum from a patient with THSD7A-associated MN or a from healthy individual into male BALB/c mice (weight, $30 \mathrm{~g}$ ). Original magnification, $\times 1,000,4 \times$ zoom (enlargement of the boxed area). (B and C) Asterisks indicate podocyte nuclei. Scale bars: $50 \mu \mathrm{m}$.

merular podocytes (16), and the ability of human anti-THSD7A autoantibodies to bind to the murine antigen, we hypothesized that human anti-THSD7A antibodies cause MN in mice. To investigate the pathogenicity of anti-THSD7A antibodies, we injected BALB/c mice with a single i.p. dose of anti-THSD7A antibodycontaining sera from patients with MN that were previously validated for the binding of mouse THSD7A (Figure 2). Thirteen mice received $\mathrm{MN}$ serum 1, seven mice received $\mathrm{MN}$ serum 2, and twelve mice received serum from a healthy individual. The glomerular binding of huIgG in mice injected with anti-THSD7A antibody-containing serum was initially found to be linear and on the subepithelial aspect of the GBM, as shown by its relation to the GBM-specific protein laminin (Figure 3A). By day 14, this linearity had changed toward a more granular pattern that became more prominent over time and persisted for at least 70 days. In contrast, mice injected with control serum failed to show binding of huIgG at the glomerular filtration barrier (Figure 3A). Notably, huIgG colocalized with THSD7A in mice that were exposed to sera from MN patients, suggesting antigen specificity of bound huIgG in subepithelial immune complexes (Figure 3B). In order to further investigate the specificity of bound huIgG in these mice, we eluted huIgG from frozen sections of kidney specimens 14 days and 70 days after injection of anti-THSD7A antibody-containing or control serum. HuIgG eluted from mice that received antiTHSD7A antibody-containing serum, but not from control mice, solely recognized a $250-\mathrm{kDa}$ protein present in both human and mouse glomerular extracts (HGEs and MGEs) as well as recombinant mouse THSD7A, confirming that the huIgG that bound in mouse glomeruli was indeed specific for THSD7A (Figure 3C). All mice that were exposed to human serum were found to have circulating mouse anti-huIgG, which was absent in mice injected with sodium chloride instead of human serum (Supplemental Figure 3). However, subepithelial deposits composed of mouse $\operatorname{IgG}(\mathrm{mIgG})$ were exclusively found in mice that received antiTHSD7A antibody-containing serum (Figure 4A). Thereby, mIgG colocalized with huIgG, suggesting the presence of subepithelial immune complexes containing THSD7A, human anti-THSD7A antibodies, and mouse anti-huIgG antibodies (Supplemental Figure 4). Mesangial huIgG and mIgG deposition was observed in all mice that were exposed to human serum. Moreover, histological analyses revealed positive staining for complement C3 in a granular subepithelial pattern on day 70 in mice that received anti-THSD7A antibody-containing serum (Figure 4B), suggesting local activation of the complement system. Immunogold electron microscopic studies demonstrated enhanced binding of an antiTHSD7A antibody to podocyte foot processes and accumulations 
A
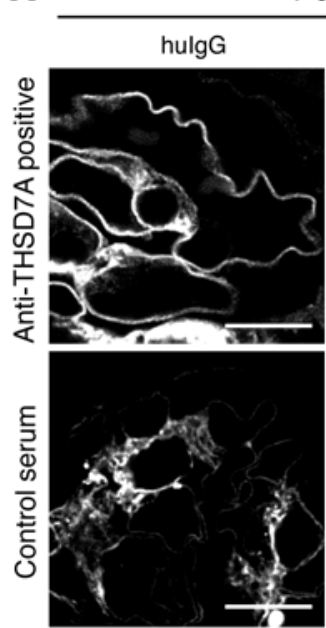

B

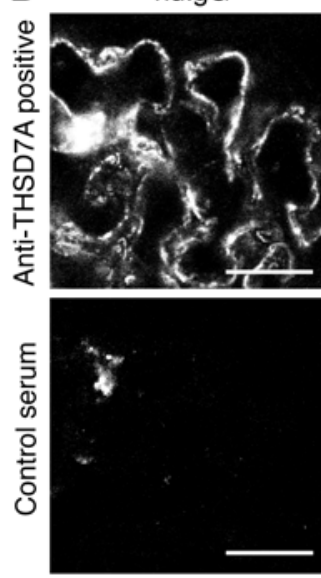

7 days
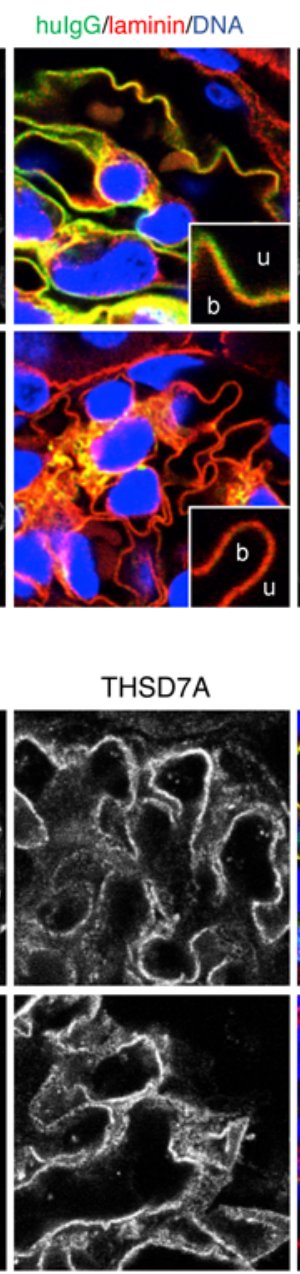

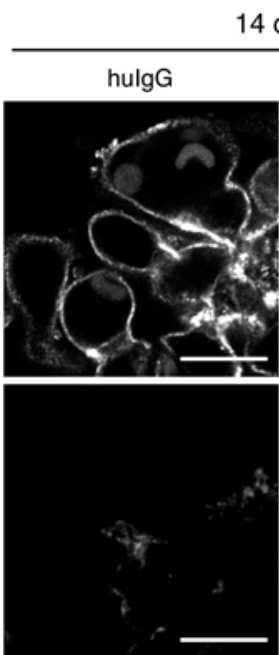

14 days

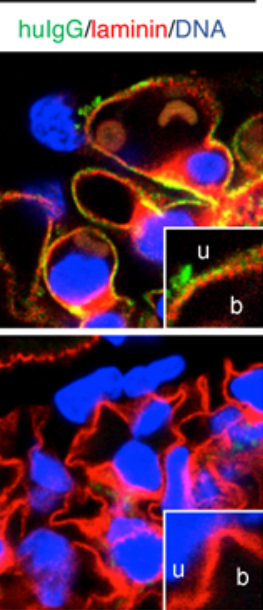

70 days

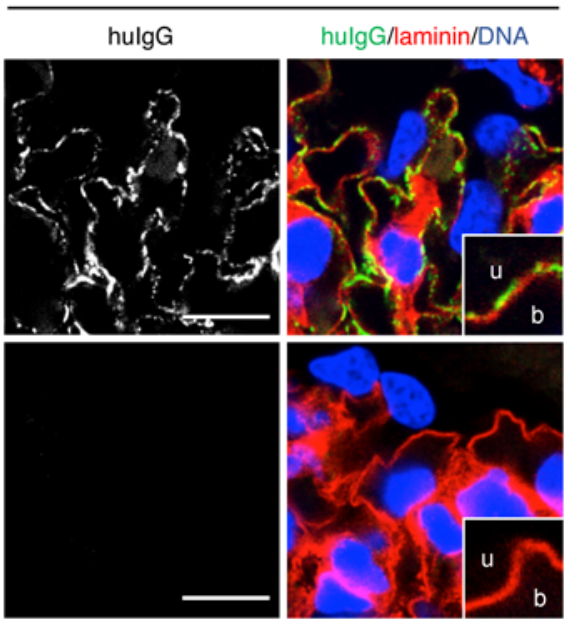

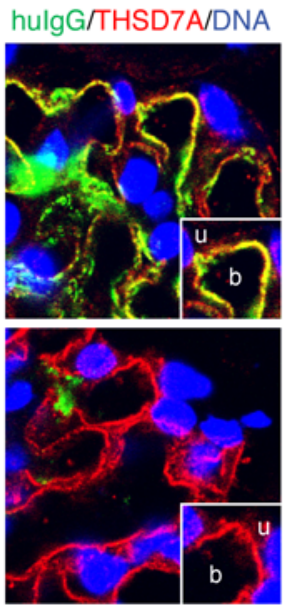

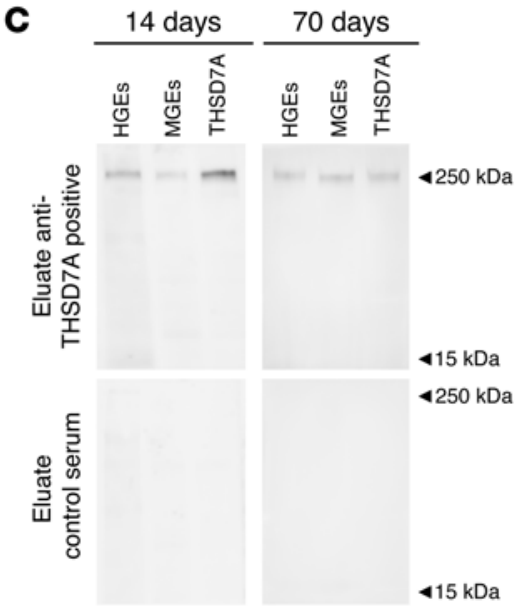

Figure 3. Anti-THSD7A antibodies induce the histological features of MN in mice. (A) Immunofluorescence staining (paraffin sections) for hulgG and laminin in mice injected with anti-THSD7A antibody-containing or control serum at different time points. b, blood side of GBM; u, urinary side of GBM. Scale bars: $10 \mu \mathrm{m}$. Enlargements are of boxed areas (original magnification, $\times 4$ ) of the glomerular filtration barrier. (B) Immunofluorescence staining (frozen sections) for hulgG and THSD7A in mice injected with anti-THSD7A antibody-containing serum or control serum. Scale bars: $10 \mu \mathrm{m}$. (C) Immunoblots of HGEs, MGEs, and recombinant mouse THSD7A with eluates from frozen kidney sections from mice that either received anti-THSD7A antibody-containing serum or control serum.

within the subepithelial aspect of the GBM, suggesting shedding of THSD7A to the subepithelial space in the mouse injected with anti-THSD7A antibody-containing serum (Figure 5A). THSD7A expression was also found on foot processes in the control mouse (Figure 5A), which was comparable in extent and localization to what was described previously (17). In immunohistochemical analyses, we found strong granular staining for huIgG along the GBM on day 70, closely resembling the morphology of human MN (Figure 5B). In line with these findings, ultrastructural studies of the same mouse demonstrated electron-dense deposits in a strictly subepithelial localization and some broadening of podocyte foot processes (Figure 5C). We also found regions with focal podocyte foot process effacement over electron-dense deposits (Supplemental Figure 5). None of these changes were seen in mice that received the control serum (Supplemental Figure 5).

Podocyte injury in $\mathrm{MN}$ is accompanied by oxidative stress and an upregulation of superoxide dismutase 2 (SOD2) (18) to detoxify
ROS. In line with this, we found SOD2 expression to be enhanced in mouse podocytes 70 days after injection of anti-THSD7A antibody-containing serum (Figure 5D). Moreover, podocyte injury was also reflected by disruption of nephrin immune localization in these mice but not in controls (Figure 5E).

Human anti-THSD7A autoantibodies induce proteinuria in mice. We examined whether the transfer of human anti-THSD7A antibody-containing serum caused proteinuria in mice. We found that around day 3, mice injected with anti-THSD7A antibody-containing serum developed significant albuminuria. The albuminuria was further increased after 14 days and remained elevated during the 70-day observation period and was supposedly induced by the autologous reaction against the persistently bound huIgG, the glomerular deposition of mouse anti-huIgG, and the subsequent activation of complement (ref. 19 and Figure 5F). Of note, albuminuria on day 3 occurred prior to enhanced glomerular binding of mIgG and deposition of complement (Supplemental Figure 6). 


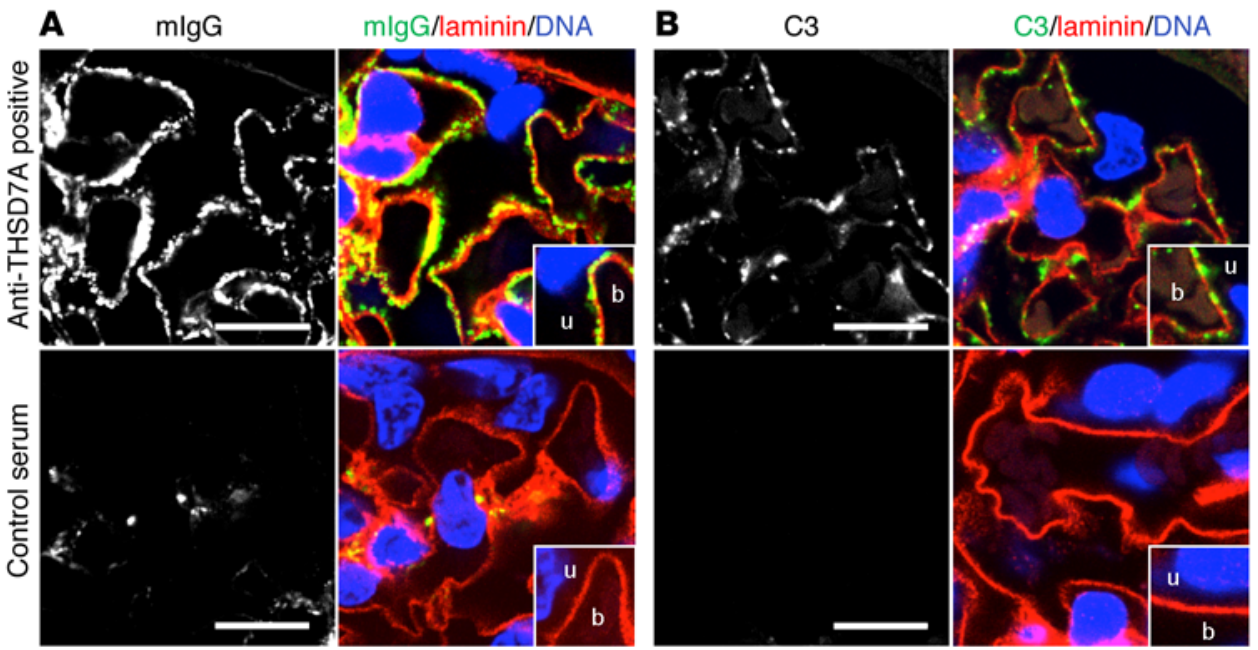

Figure 4. Mouse IgC and complement C3 are deposited in the glomeruli of mice that were injected with antiTHSD7A-containing serum. (A and B) Immunofluorescence staining (paraffin sections) for mlgG and laminin (A) and complement component $\mathrm{C} 3$ and laminin (B) on day 70 . Scale bars: $10 \mu \mathrm{m}$
Urinary albumin excretion in mice injected with serum from a healthy donor remained unchanged (Figure 5F).

Affinity-purified THSD7A-specific huIgG causes the histological features of $M N$ and transient proteinuria. In order to investigate whether it is indeed the serum autoantibodies against THSD7A that are causative for MN, we affinity purified THSD7A-specific huIgG from anti-THSD7A antibody-containing serum using chromatographic columns containing beaded agarose coupled to DDKpurified recombinant THSD7A. Three rounds of purification were performed, with a total of six acid elutions, reducing the content of anti-THSD7A antibodies in the serum to nondetectable levels (Figure 6A). Subsequently, 4 mice each were injected with antiTHSD7A antibody-depleted serum or with affinity-purified antiTHSD7A antibodies from the pooled eluates. Morphological analyses demonstrated granular subepithelial deposition of purified anti-THSD7A antibodies, whereas solely mesangial huIgG deposition was observed in mice injected with the depleted serum (Figure 6B). Again, huIgG colocalized with THSD7A in immune deposits (Figure 6C) and induced a granular subepithelial mIgG deposition (Figure 6D) only in mice injected with purified anti-THSD7A antibodies. Altogether, the subepithelial deposition of huIgG and mIgG was less pronounced in mice injected with purified anti-THSD7A antibodies than in mice that received anti-THSD7A antibody-containing whole serum (Figure 3), a finding that was likely related to antibody loss during the purification procedure.

Clinically, injection of purified anti-THSD7A antibodies induced significant albuminuria after 3 days when compared with mice that received anti-THSD7A antibody-depleted serum (Figure $6 \mathrm{E})$. After 3 weeks, proteinuria gradually decreased to the level seen in depleted serum-injected mice. No complement C3 deposition was detected in any of the mice (data not shown), suggesting that the amount of injected purified anti-THSD7A antibodies was not sufficient to induce an autologous phase with maintenance of proteinuria. Taken together, these experiments demonstrate that anti-THSD7A antibodies alone are sufficient to induce MN.

Anti-THSD7A autoantibodies cause cytoskeletal rearrangement in primary cultured glomerular epithelial and human embryonic kidney 293 cells. We next examined whether anti-THSD7A antibodies can directly affect the cellular architecture of THSD7A-expressing cells. Immortalized cultured murine or human podocyte cell lines do not express THSD7A at detectable protein levels (our unpublished data). We therefore generated primary glomerular epithelial cells (GECs) by culturing isolated murine glomeruli for 3 to 5 days on collagen 1-coated plates. Western blot analysis showed THSD7A expression in GECs (Figure 7A). Exposure of GECs to affinity-purified anti-THSD7A antibodies resulted in binding of huIgG to the cell membrane. Cells with membrane-bound huIgG stained positive for the standard podocyte marker protein $\alpha$-actinin-4, suggesting that these cells were indeed podocytes (Figure 7B). Cells expressing $\alpha$-actinin- 4 that were treated with serum depleted of anti-THSD7A antibodies or with huIgG that was purified from a healthy donor failed to bind huIgG (Figure 7B and Supplemental Figure 7). Importantly, cells that did not stain for $\alpha$-actinin-4 also did not bind huIgG following incubation with affinity-purified anti-THSD7A antibodies (arrows in Figure 7B), indicating that the binding of THSD7A autoantibodies was specific for mouse primary podocytes.

GECs that exhibited huIgG binding following exposure to affinity-purified anti-THSD7A antibodies showed marked cytoskeletal rearrangement, with formation of stress fibers and strong enhancement of F-actin staining (Figure 7C). When quantifying F-actin OD, we found a 2.5-fold increase in cells treated with purified anti-THSD7A antibodies as compared with that detected in cells treated with depleted serum (Figure 7D). Similar results were obtained when anti-THSD7A antibody-containing whole serum was used for experimental procedures (Supplemental Figure 8).

Focal adhesions are highly organized multiprotein assemblies that mediate signaling between the actin cytoskeleton and the extracellular matrix (20). Paxillin is a vital member of focal adhesions (21) and connects integrin signaling with cytoskeletal remodeling (22). In order to investigate whether the changes in cytoskeletal organization were accompanied by altered focal adhesions, we performed staining for phosphorylated paxillin (p-paxillin). We found an increase in focal adhesions with positive p-paxillin staining in GECs that were exposed to purified anti-THSD7A antibodies, but not in GECs treated with depleted serum (Figure 7C). Moreover, p-paxillin was shifted from the leading edges of cells toward cell centers, according to the formation of stress fibers in 
A

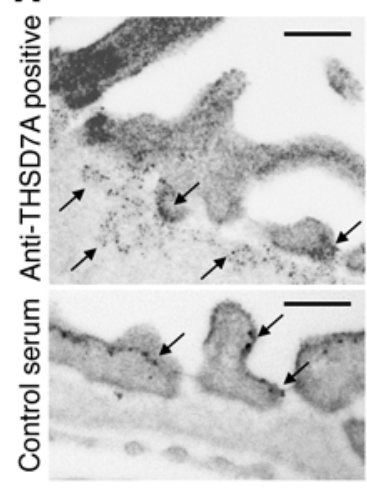

D

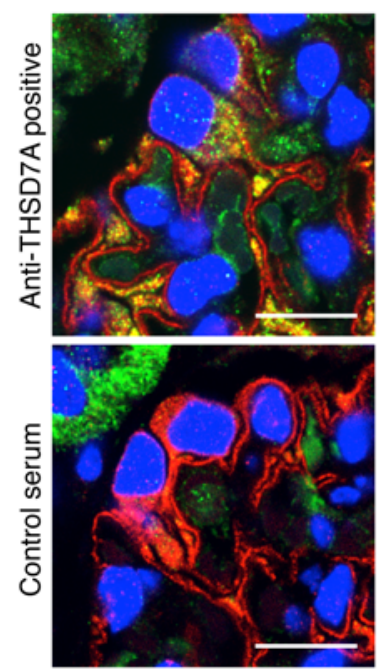

SOD2/nephrin/DNA
B

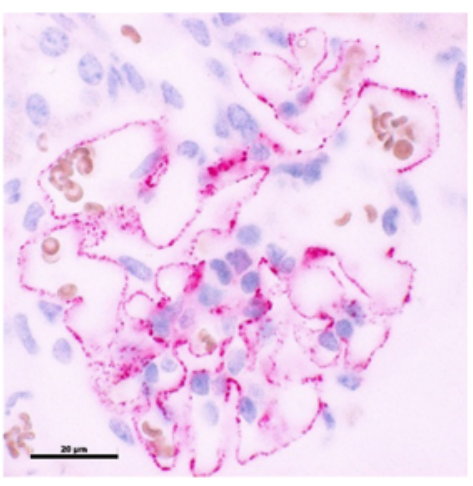

E
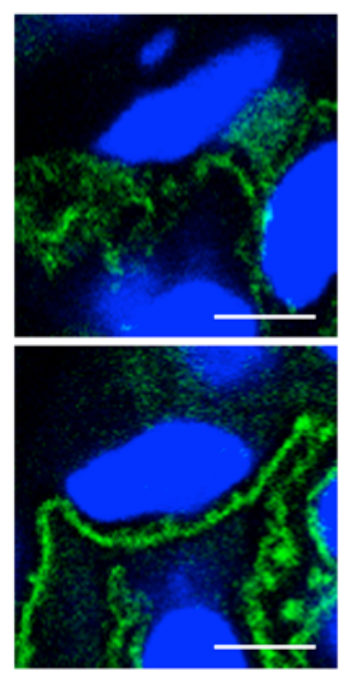

Nephrin/DNA

$\mathbf{F}$
C
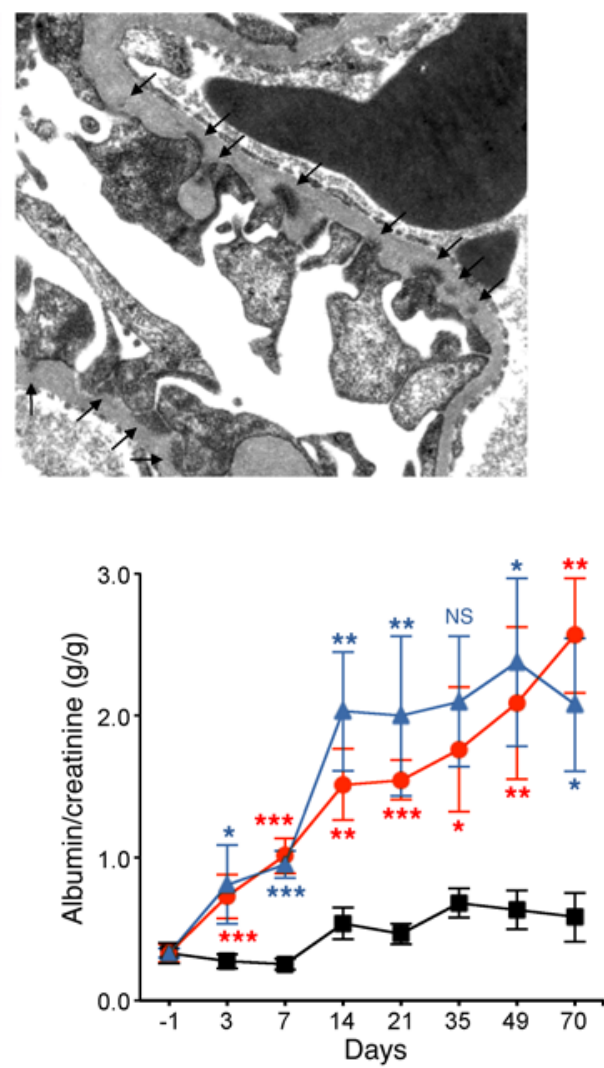

No. of animals

$\begin{array}{lcccccccc}- \text { - Control serum } & 12 & 12 & 11 & 10 & 9 & 8 & 8 & 7 \\ - \text { MN 1 } & 13 & 13 & 12 & 11 & 10 & 9 & 8 & 6 \\ - \text { MN2 } & 7 & 7 & 7 & 6 & 5 & 5 & 5 & 5\end{array}$

Figure 5. Anti-THSD7A antibodies induce ultrastructural features of MN and proteinuria in mice. (A) Immunogold electron microscopic images of THSD7A expression in mice after injection of anti-THSD7A antibody-containing or control serum using a specific anti-THSD7A antibody and 12-nm gold-coupled anti-rabbit antibodies on day 70 . Scale bars: $200 \mathrm{~nm}$. (B) Immunohistochemical staining for hulgC on paraffin-embedded tissue sections from a mouse 70 days after injection of anti-THSD7A antibody-containing serum. Scale bar: $20 \mu \mathrm{m}$. (C) Electron microscopic image from the same mouse 70 days after injection of anti-THSD7A antibody-containing serum. Arrows indicate subepithelial electron-dense deposits. Original magnification, $\times 12,500$. (D and E) Immunofluorescence staining for SOD2 (D) and nephrin (E) on paraffin sections of kidney samples from mice that received either anti-THSD7A antibody-containing serum or control serum on day 70 . Scale bars: $10 \mu \mathrm{m}$ (D) and $5 \mu \mathrm{m}$ (E). (F) Albumin-to-creatinine ratios measured in urine samples from mice injected with either anti-THSD7A antibody-containing serum or control serum. Error bars indicate the mean $\pm \mathrm{SEM}$. ${ }^{*} P<0.05$, ${ }^{* *} P<0.01$, a nd ${ }^{* * *} P<0.001$, by 2-tailed, nonparametric Mann-Whitney $U$ test of MN 1 or MN 2 versus control serum.

cells with bound huIgG, but not in cells devoid of huIgG binding (Figure 7C, arrows).

Furthermore, GECs exhibited an elongated morphology following exposure to anti-THSD7A antibody-containing serum (Supplemental Figure 9), and some detached from the culture plates and were lost to the medium (data not shown). Since GECs are a heterogeneous population of glomerular cells, we repeated the experiments in human embryonic kidney 293 (HEK293) cells for adequate quantification of the observed effects. HEK293 cells transiently transfected with human THSD7A or empty vector (Figure 8A and Supplemental Figure 10) were incubated with medium containing $2 \%$ of the anti-THSD7A antibody-containing or control serum. When incubated with anti-THSD7A antibody-containing serum, binding of huIgG on the cell membrane and predominantly at cell-cell junctions of THSD7A-expressing HEK293 cells and colocalization of huIgG with THSD7A were detected (Sup- plemental Figure 10). Following exposure to anti-THSD7A antibody-containing serum, confluent THSD7A-expressing cells significantly detached from their culture plates (Supplemental Figure 11A) and were lost to the culture medium (Figure 8B). FACS-based analyses demonstrated that detached cells were THSD7A positive and had huIgG bound to the cell membrane (Supplemental Figure 11B). Additionally, detached cells with bound huIgG were alive, as demonstrated by exclusion of ViViD staining in THSD7A-expressing cells (Supplemental Figure 11C). THSD7A-expressing HEK293 cells did not detach from the culture plates when incubated with control serum, serum from patients with $\mathrm{PLA}_{2} \mathrm{R} 1$-associated $\mathrm{MN}$, or serum from patients with $\mathrm{MN}$ that had neither anti-THSD7A nor anti-PLA 2 R1 autoantibodies (Figure 8B). In contrast, cells significantly detached when incubated with affinity-purified antiTHSD7A antibodies, but not with anti-THSD7A-depleted serum or purified control IgG (Figure $8 \mathrm{C}$ ). 
A

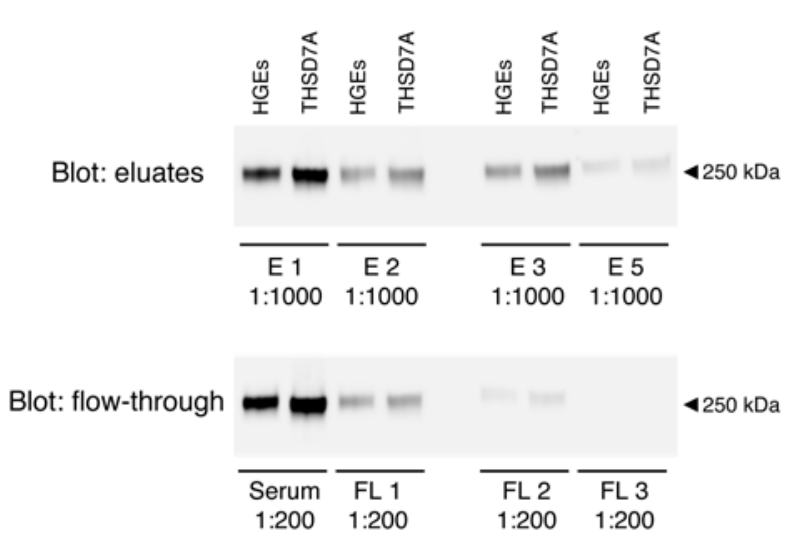

B

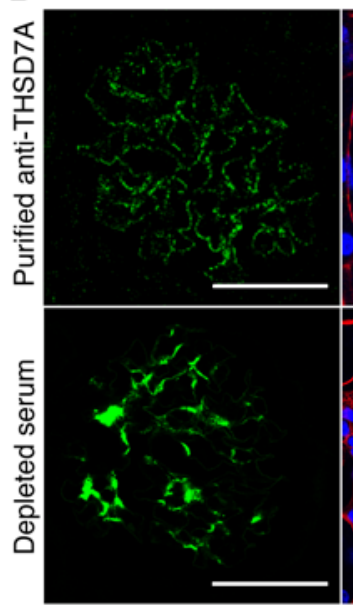

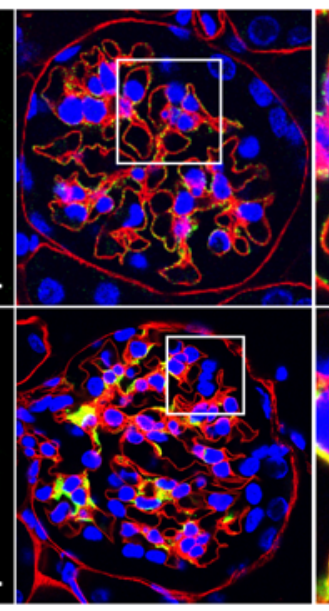

hulgG/laminin/DNA

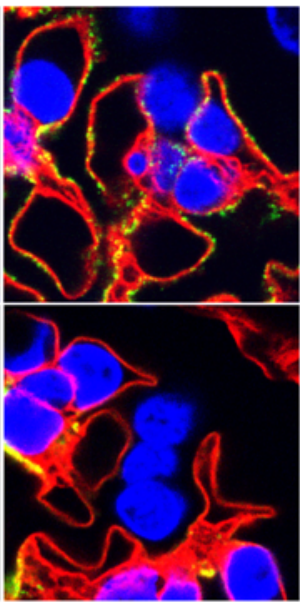

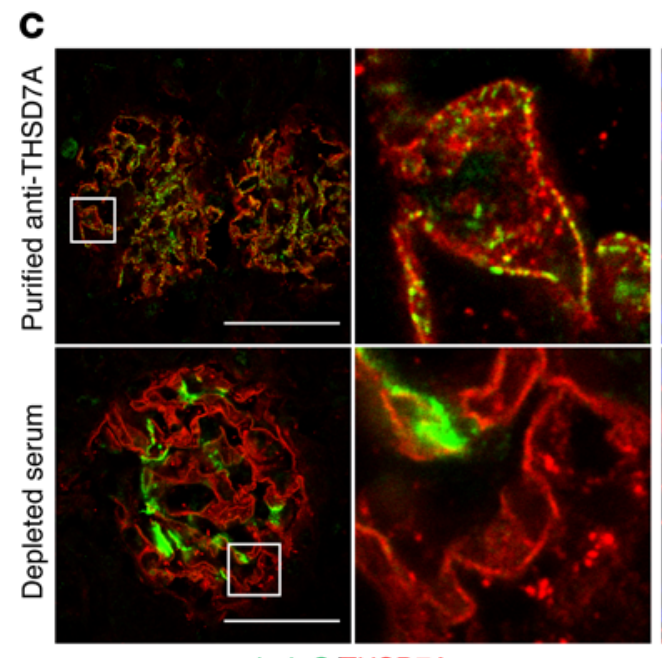

hulgG/THSD7A
D

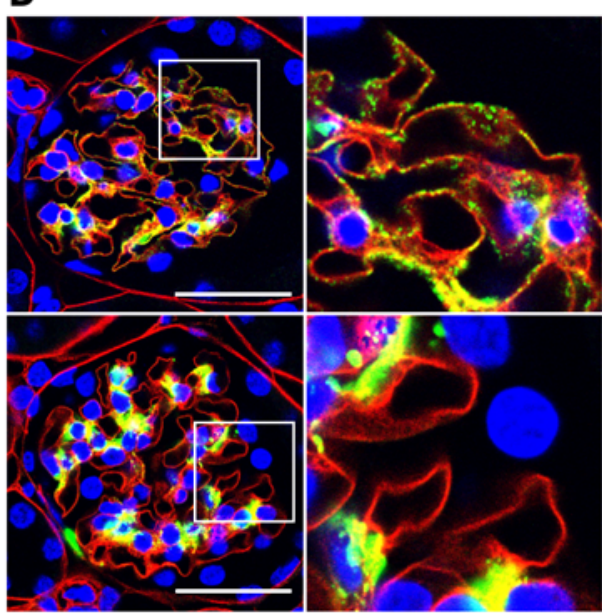

mlgG/laminin/DNA
E

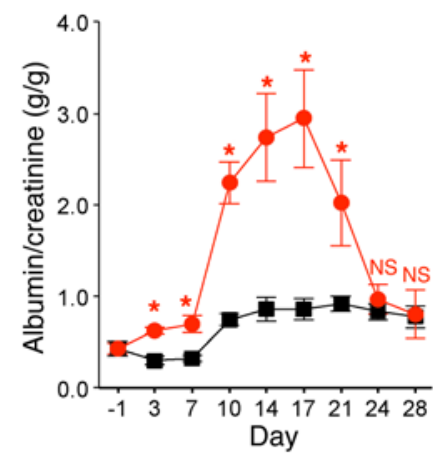

- Depleted serum

Purified anti-THSD7A

Figure 6. Affinity-purified anti-THSD7A antibodies induce MN in mice. (A) Serum from a patient with THSD7A-associated MN was run through chromatographic columns loaded with beaded agarose that was coupled to purified recombinant human THSD7A. The serum was passed 3 times over the columns (FL1-FL3) with 2 acid elutions per round (E1-E6). Immunoblots show the reactivity of purified anti-THSD7A antibodies and serum flow-through with HGEs and recombinant human THSD7A. (B-D) Immunofluorescence staining for hulgG and laminin (paraffin sections) (B), hulgG and THSD7A (frozen sections) (C), and mlgC and laminin (paraffin sections) (D) 28 days after injection of purified anti-THSD7A antibodies or of serum depleted of antiTHSD7A antibodies. Images are representative of analyses of 4 animals per group. Scale bars: $50 \mu \mathrm{m}$. Enlargements of boxed areas in B-D are shown in the far right upper and lower panels (original magnification, $\times 1,000 ; 4 \times z 00 \mathrm{~m}$ ). (E) Albumin-to-creatinine ratios measured in urine samples from mice injected with either purified anti-THSD7A antibodies or depleted serum ( $n=4$ per group). Error bars indicate the mean \pm SEM. ${ }^{*} P<0.05$, by 2 -tailed, nonparametric Mann-Whitney $U$ test.

In line with these observations, incubation with anti-THSD7A antibody-containing serum and purified anti-THSD7A antibodies resulted in the cytoskeletal rearrangement of THSD7A-expressing cells that was characterized by strong enhancement of cortical F-actin fibers (Figure 8D and Supplemental Figure 12). Taken together, these results suggest a direct influence of anti-THSD7A antibodies on cytoskeletal organization of THSD7A-expressing GECs and HEK293 cells.

\section{Discussion}

A number of glomerular proteins have been identified as potential targets for autoimmunity in $\mathrm{MN}$ in recent years. These molecules include the podocyte membrane proteins NEP (6) and
$\mathrm{PLA}_{2} \mathrm{R} 1$ (7). Additionally, other intracellular antigens such as SOD2, ubiquitin C-terminal hydrolase L1 (UCHL1), and aldose reductase have been described (18). However, the question of whether autoantibodies against all these antigens are actually causing the morphological and clinical picture of MN has intrigued researchers in the field for years (23). The recent discovery of THSD7A as another endogenously expressed podocyte antigen in human MN (8) and the fact that THSD7A is expressed in mouse podocytes $(16,17)$ with more than $90 \%$ sequence homology to the human molecule have given rise to experimental approaches in the investigation of MN pathogenesis.

In light of our observation of a patient who presented with a recurrence of THSD7A-associated MN in a renal transplant in 
A

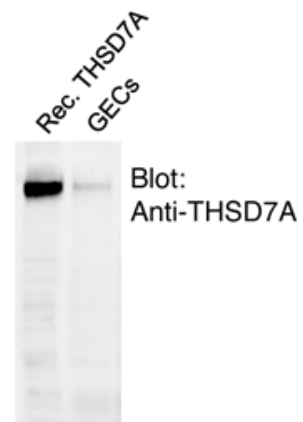

B

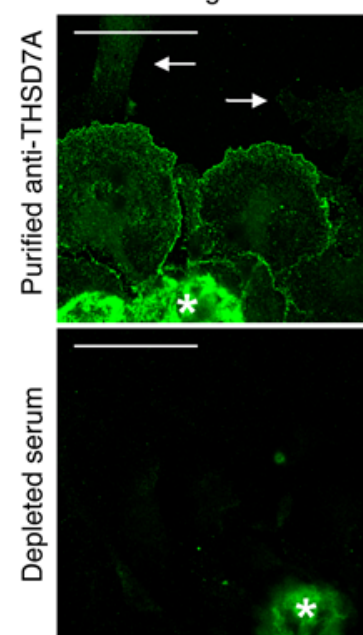

$\alpha$-Actinin-4

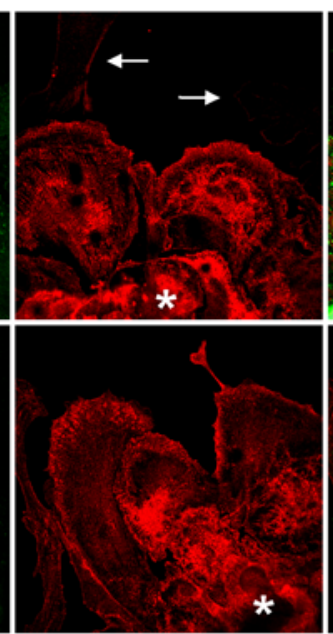

hulgG/ $\alpha$-actinin-4/DNA

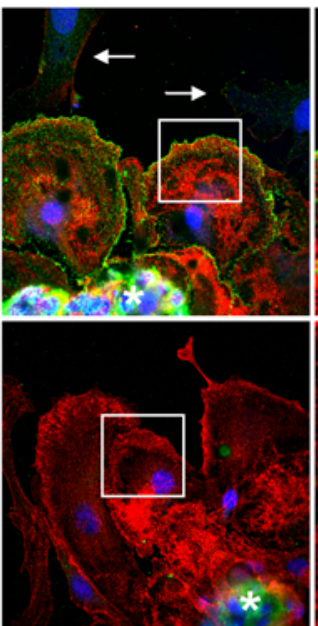

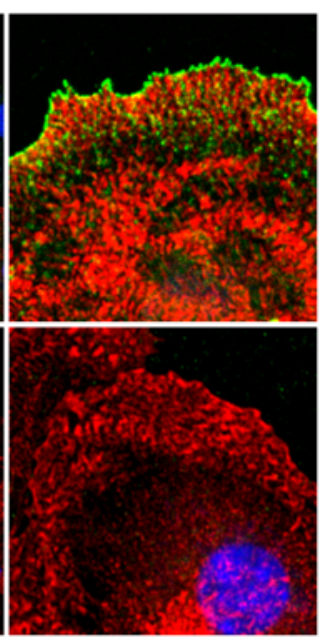

C

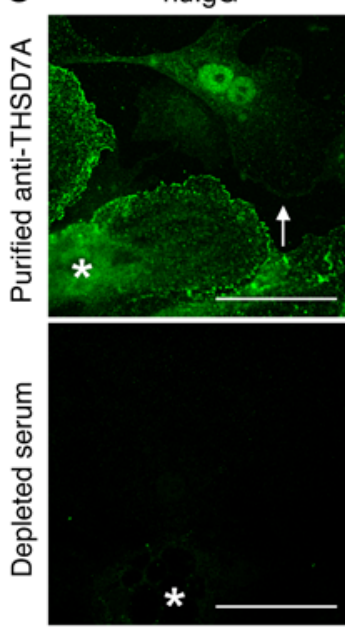

F-actin

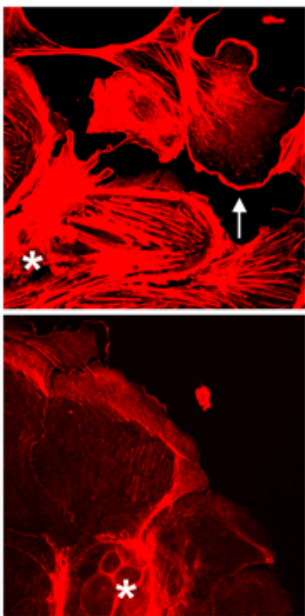

p-Paxilin

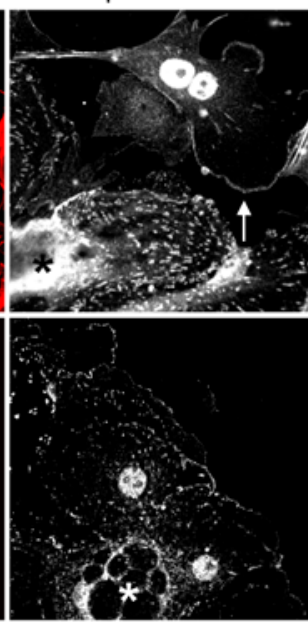

Merge

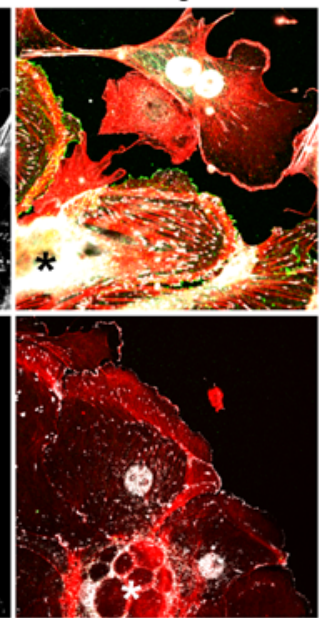

D



Figure 7. Anti-THSD7A antibodies cause cytoskeletal rearrangement in THSD7A- expressing primary cultured GECs. (A) Western blot analysis shows expression of THSD7A in GECs. Rec. THSD7A, recombinant human THSD7A. (B) Immunofluorescence staining for hulgG and $\alpha$-actinin-4 in GECs following a 40-minute exposure to affinity-purified anti-THSD7A antibodies or to serum that was depleted of anti-THSD7A antibodies. Arrows indicate cells that did not express $\alpha$-actinin- 4 and that showed no hulgG binding. Asterisks indicate the glomerulus from which the cells grew. Scale bars: $50 \mu \mathrm{m}$. Enlargements of boxed areas are shown in the far right upper and lower panels (original magnification, $\times 1,000,4 \times$ zoom). (C) Immunofluorescence staining for hulgG, F-actin (phalloidin), and p-paxillin in GECs following a 40-minute exposure to affinity-purified anti-THSD7A antibodies or to serum that was depleted of anti-THSD7A antibodies. Arrows indicate a cell that had no hulgC bound to the membrane and that did not exhibit enhanced F-actin staining. Asterisks indicate the glomerulus from which the cells grew. Scale bars: $50 \mu \mathrm{m}$. (D) Quantification of stress fiber formation after treatment of GECs with purified anti-THSD7A antibodies or serum depleted of anti-THSD7A antibodies. A total of 30 images from 3 independent experiments were analyzed. Data indicate F-actin OD of individually circled cells normalized to the control condition (depleted serum). Error bars represent the mean \pm SEM. ${ }^{* * *} P<0.001$, by 2 -tailed, nonparametric Mann-Whitney $U$ test.

the presence of circulating anti-THSD7A antibodies, we hypothesized that anti-THSD7A antibodies could be causative for MN in humans. We therefore initiated studies in mice, transferring anti-THSD7A antibodies from 2 patients with THSD7A-associated MN. Our data demonstrate that human anti-THSD7A antibodies can bind to the murine antigen in vivo and in vitro. Moreover, the administration of both anti-THSD7A antibody-containing serum and isolated THSD7A-specific IgG to mice led to the development of proteinuria and a histomorphological pattern of MN. Injected antibodies thereby reached the glomerulus, bound to their target antigen, and induced subepithelial immune deposits containing THSD7A and anti-THSD7A antibodies. The binding of
anti-THSD7A antibodies to THSD7A resulted in the onset of proteinuria, establishing the causal relationship between podocytedirected autoantibodies and the development of MN.

That this concept is also operative in human $\mathrm{MN}$ was first shown in 2002, when Debiec et al. discovered autoantibodies against NEP to be associated with a rare form of newborn alloimmune MN (6). They could also demonstrate that injection of whole IgG isolated from the mother (who gave birth to a child with antenatal NEP-associated MN) into rabbits induced proteinuria and glomerular immune complexes (6). In our experiments, both whole serum and affinity-purified THSD7A-specific IgG induced proteinuria within 3 days after administration to mice. In the ani- 
A

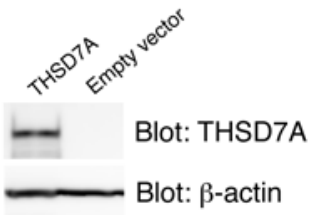

B

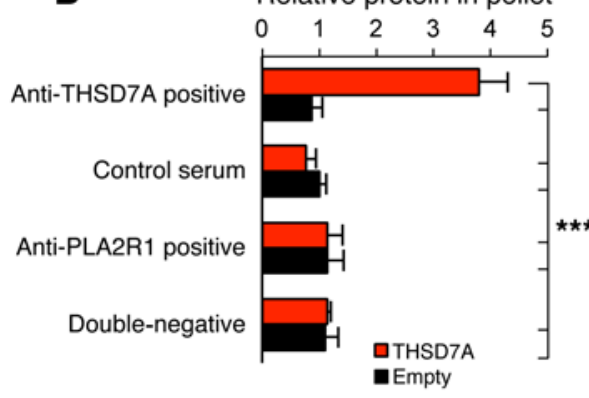

C

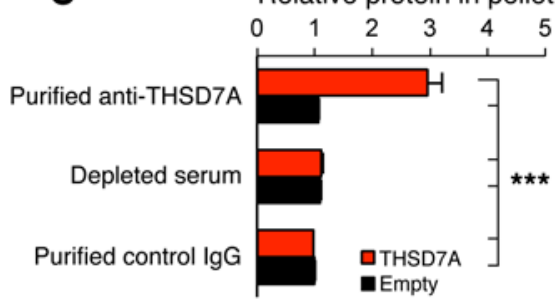

D

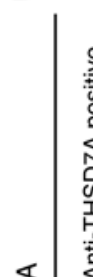

hulgG

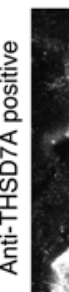

F-actin


Figure 8. Anti-THSD7A antibodies induce cell detachment and cytoskeletal rearrangement in THSD7A-expressing HEK293 cells. (A) Western blot analysis shows reactivity of a specific anti-THSD7A antibody in HEK293 cells that were transfected with THSD7A cDNA or empty vector. (B and C) Quantification of detached cells by measurement of total protein concentration in cell pellets after centrifugation of cell culture supernatants following a 60-minute incubation with (B) anti-THSD7A antibody-containing or control serum (results are from at least 5 independent experiments with 2 anti-THSD7 antibody-positive sera, 5 anti-PLA $A_{2}$ 1 antibody-positive sera, 5 sera with no antibodies against THSD7A or PLA R1, and 1 control serum) or with (C) affinity-purified antiTHSD7A antibodies, depleted serum, and affinity-purified IgC from a healthy individual (results are from 5 independent experiments with antibodies purified from 1 patient, the corresponding depleted serum, and purified control IgG). Data indicate the fold change in protein content in the cell culture supernatant in comparison with the cells that were transfected with empty vector and treated with serum from a healthy donor (B) or with purified control lgG (C). Error bars indicate the mean $\pm \mathrm{SEM}$. ${ }^{* *} P<0.001$, by 1-way ANOVA with Bonferroni's post test; statistical significance is shown for all conditions versus THSD7A-transfected cells that were treated with anti-THSD7A antibody-containing serum (B) or affinity-purified anti-THSD7A antibodies (C). (D) Immunofluorescence staining for hulgG and F-actin (phalloidin) in empty vector- and THSD7A-transfected HEK293 cells after a 30-minute exposure to anti-THSD7A antibody-containing serum or control serum. Arrows show accentuated cortical F-actin rings; asterisks indicate detaching cells. Scale bars: $20 \mu \mathrm{m}$.

mals receiving whole serum, we could detect large amounts of mouse anti-huIgG antibodies at later time points, suggesting that the bound huIgG served as a neoantigen in the glomerular filtration barrier, thereby inducing an autologous phase of the disease. In line with this, proteinuria persisted for the entire observation period in the animals injected with whole serum.

In contrast, proteinuria in animals that received the purified anti-THSD7A antibodies was transient, which we cannot sufficiently explain at this point. However, some issues may be relevant. There is considerable evidence from studies in passive Heymann nephritis that proteinuria in this model depends on activation of the complement system and that the complement membrane attack complex C5b-9 is responsible for podocyte injury and the development of proteinuria $(5,24)$. Other studies suggest that proteinuria can develop in the absence of certain complement factors $(25,26)$. We therefore analyzed mouse kidneys from our experiments for the presence of complement components in the subepithelial immune deposits. In the early phase of the disease, we could not detect $\mathrm{C} 3$ or C5b-9 in mice that received whole serum or in mice that received purified anti-THSD7A antibodies alone. Later in the course, marked $\mathrm{C} 3$ deposits were found in the mice injected with whole serum, but were absent in the animals receiving anti-THSD7A antibodies alone. Thus, it is possible that at early time points, complement activation is not vital to the initiation of podocyte injury and proteinuria in this model, but that at later time points, when larger amounts of autologous mouse anti- 
bodies bind to huIgG, complement gets activated and maintains the disease. Also, the acid-eluted heterologous huIgG alone might be incapable of inducing or insufficient to induce the mouse complement system, with complement activation in this case entirely relying on the presence of large amounts of mIgG.

Several studies describe direct effects of antibodies on molecular processes that are potentially relevant in the pathogenesis of MN. In the rat model of Heymann nephritis, anti-megalin antibodies directly affect podocyte function by preventing the uptake of apolipoproteins through megalin, resulting in apolipoprotein deposition in immune deposits (27). In alloimmune antenatal MN, different anti-NEP antibody subclasses have different potencies in inhibiting enzymatic NEP activity in vitro (9). Moreover, in a case of IgG4-related disease that was associated with MN, it was demonstrated that the binding of circulating IgG4 anti-carbonic anhydrase II antibodies to the membrane of cultured podocytes induced a podocyte stress reaction with externalization of SOD2 (28). Given the surprising result that mice developed significant proteinuria in the absence of complement activation in the first week after administration of the antibodies, we aimed to investigate whether anti-THSD7A antibodies can directly affect podocyte integrity. We found that THSD7A-expressing GECs underwent marked cytoskeletal rearrangement, with an alteration in cellular morphology and focal adhesions upon binding of the antibody to the cell membrane. Moreover, anti-THSD7A antibodies caused cell detachment and enhanced F-actin staining in HEK293 cells that were transiently transfected with THSD7A. These in vitro results indicate that antiTHSD7A antibodies might directly interfere with podocyte integrity in vivo as well, leading to cell damage and proteinuria.

In summary, the present study describes a patient with THSD7A-associated MN, whose rapid recurrence of MN after kidney transplantation in the presence of anti-THSD7A antibodies suggests a causative role of these antibodies in disease onset. We translated this clinical observation into animal studies and demonstrated that anti-THSD7A autoantibodies from patients with THSD7A-associated MN bound to podocyte-expressed THSD7A on foot processes in mice and induced proteinuria and the morphologic hallmarks of $\mathrm{MN}$. The glomerular binding of huIgG was thereby THSD7A specific, demonstrating that it is in fact this antigen-antibody interaction that induced MN pathology in mice. We believe that our study uncovers the causative role of autoantibodies in the development of MN and supports the pathogenic concept of this disease.

\section{Methods}

Patients. The diagnosis of MN was made by renal biopsy. For animal experiments, sera were taken from 2 patients with THSD7A-associated $\mathrm{MN}$ and from healthy controls. All patients were without previous immunosuppressive therapy at the time of serum collection. Sera were investigated for anti-PLA 2 R1 autoantibodies by means of ELISA (29). Anti-THSD7A antibodies were detected using Western blotting, and titers were quantified by indirect immunofluorescence testing (IFT) as described previously for PLA 2 R1 (30) (test was provided by EUROIMMUN AG, Lübeck, Germany).

Immunohistochemical analyses. Paraffin sections $(1-\mu \mathrm{m})$ of renal biopsies from a 57-year-old patient with $\mathrm{MN}$ and a healthy control patient were deparaffinized and rehydrated. Antigen retrieval was obtained for THSD7A by boiling in Dako antigen retrieval buffer, $\mathrm{pH}$ $9\left(15\right.$ minutes at $\left.98^{\circ} \mathrm{C}\right)$; for $\mathrm{C} 4 \mathrm{~d}$ by boiling in citrate buffer, $\mathrm{pH} 6.0$ (30 minutes at $\left.98^{\circ} \mathrm{C}\right)$; and for huIgG by protease XXIV treatment $(5 \mu \mathrm{g} / \mathrm{ml}$ at $37^{\circ} \mathrm{C}$ for 15 minutes; Sigma-Aldrich). Nonspecific binding was blocked with $5 \%$ horse serum (Vector Laboratories) with $0.05 \%$ Triton X-100 (Sigma-Aldrich) in PBS for 30 minutes at room temperature (RT) prior to incubation at $4^{\circ} \mathrm{C}$ overnight with rabbit anti-THSD7A (1:400; Atlas; catalog HPA000923); anti-C4d (1:200; BIOZOL; catalog BI-RC4D); or anti-huIgG (1:200, Cy2-huIgG H+L; Dianova; catalog 709-225-149) in blocking buffer. Staining was visualized with a ZytochemPlus AP Polymer Kit (Zytomed Systems) according to the manufacturer's instruction. Nuclei were counterstained with hemalaun, and sections were mounted with gum arabic (Sigma-Aldrich). Negative controls were performed by omitting primary antibodies. Stainings were evaluated with an Axioskop using the Axiovision software (both from Zeiss).

Animal care. WT male BALB/c mice (10- to 14-weeks of age) were bred in the animal facility at the University Medical Center HamburgEppendorf. Animals had free access to water and standard animal chow.

Animal experiments. The pathogenicity of anti-THSD7A antibodies was evaluated in 2 experimental setups. In the first setup, anti-THSD7A antibody-positive sera from 2 patients with THSD7A-associated MN were used for disease induction, while serum from a healthy donor served as a negative control. In the second approach, affinity-purified anti-THSD7A antibodies and the remaining serum that was depleted of anti-THSD7A antibodies were used. Prior to injection, human sera were concentrated 2-fold using Amicon Ultra-15 centrifugal filters with a molecular cut-off of $100 \mathrm{kDa}$ (EMD Millipore). Sera were then decomplemented by heating for 30 minutes at $56^{\circ} \mathrm{C}$ in a water bath. Debris was removed by centrifugation at $20,817 \mathrm{~g}$ for 15 minutes. As the sera from the 2 nephrotic patients contained subnormal levels of total proteins, the huIgG serum levels were quantified by SDS-PAGE and adjusted to equal levels. BALB/c mice were injected i.v. with $100 \mu \mathrm{l}$ of adjusted sera for analysis after 2 hours and i.p. with $900 \mu$ l of adjusted sera for disease induction. Development of proteinuria was monitored using metabolic cages every 3 to 4 days for 2 weeks and then weekly. The histological images presented in the figures represent analyses of mice that were sacrificed at different time points ( 2 animals after 3 days, 3 animals after 7 days, 3 animals after 14 days, and 9 animals after 70 days). For the second experimental setup, anti-THSD7A antibodies were purified from $10 \mathrm{ml}$ serum from a patient with THSD7A-associated $\mathrm{MN}$ and concentrated using Amicon Ultra-15 centrifugal filters with a molecular cut-off of $100 \mathrm{kDa}$ to a final volume of $1 \mathrm{ml}$. Four male BALB/c mice were then i.v. injected with $250 \mu$ affinity-purified anti-THSD7A antibodies. The remaining $8 \mathrm{ml}$ of depleted serum was concentrated using Amicon Ultra-15 centrifugal filters with a molecular cut-off of $100 \mathrm{kDa}$ to a final volume of $4 \mathrm{ml}$. Four male BALB/c mice were then i.p. injected with $1 \mathrm{ml}$ of depleted serum. Development of proteinuria was monitored as described above.

Immunofluorescence analyses. For immunolocalization of nephrin (guinea pig pAB, 1:100; Acris; catalog BP5030); laminin (rabbit pAB, 1:1,000; Sigma-Aldrich; catalog L9393); huIgG (Cy2 huIgG H+L, 1:200; Dianova; catalog 709-225-149); murine IgG (H+L Cy2 mIgG, 1:400; Dianova; catalog 715-225-151); complement C3 (FITC goat pAB, 1:100; Cappel; catalog 55500); or SOD2 (rabbit pAB, 1:100; Acris; catalog AP03023PU-S), 2- $\mu \mathrm{m}$ paraffin sections of normal or experimental mouse kidneys were deparaffinized and rehydrated with water. Antigen retrieval was obtained by boiling in citrate buffer, $\mathrm{pH}$ 
6.1 (both 30 minutes at a constant temperature of $98^{\circ} \mathrm{C}$ ) or by digestion with protease XXIV ( $5 \mu \mathrm{g} / \mathrm{ml}$; Sigma-Aldrich) for 15 minutes at $37^{\circ} \mathrm{C}$. Unspecific binding was blocked with $5 \%$ horse serum (Vector Laboratories) with $0.05 \%$ Triton X-100 (Sigma-Aldrich) in PBS for $30 \mathrm{~min}$ utes at RT prior to incubation at $4^{\circ} \mathrm{C}$ overnight with primary antibodies in blocking buffer. Staining was visualized with fluorochrome-conjugated secondary antibodies (1:400; all affinity purified from Jackson Immunoresearch Laboratories) for 30 minutes RT in 5\% horse serum with $0.05 \%$ Triton X-100. Nuclei were counterstained with DRAQ5 (1:1,000; Thermo Scientific; catalog 62252).

For indirect immunofluorescence using anti-THSD7A antibodypositive sera or healthy control sera, $5-\mu \mathrm{m}$ cryosections were fixed with ice-cold $100 \%$ acetone for 10 minutes at $-20^{\circ} \mathrm{C}$. Unspecific binding was blocked with $5 \%$ normal horse serum containing $0.05 \%$ Triton X-100 for 30 minutes at RT. Sera were diluted at 1:250 and incubated overnight at $4^{\circ} \mathrm{C}$ in blocking buffer concomitantly with antinephrin antibody (1:100). Autoantibody binding was visualized using Cy2 anti-huIgG, and nephrin was visualized using Cy3 anti-guinea pig (1:200; Dianova; catalog 706-165-148) for 30 minutes in blocking buffer. Nuclei were counterstained with DRAQ5 (1:1,000).

For visualization of autoantibody binding following i.v. injection of sera, $5-\mu \mathrm{m}$ cryosections were fixed with ice-cold $100 \%$ acetone for 10 minutes at $-20^{\circ} \mathrm{C}$. Unspecific binding was blocked with $5 \%$ normal horse serum containing $0.05 \%$ Triton X-100 for 30 minutes at RT. Autoantibody binding was visualized using huIgG4 (mouse mAB, 1:1,000; Binding Site; catalog MC011) incubated overnight at $4^{\circ} \mathrm{C}$ in blocking buffer concomitantly with anti-nephrin antibody (1:100). Bound autoantibodies were visualized using Cy2 anti-mouse IgG and nephrin using Cy3 anti-guinea pig for 30 minutes in blocking buffer. Nuclei were counterstained with DRAQ5 (1:1,000).

All negative controls were performed by omitting primary antibodies. Stainings were evaluated with a confocal LSM 510 meta microscope and LSM software (both from Zeiss).

Generation of antibodies against native human THSD7A. A rabbit antiserum (K1405) recognizing native human and mouse THSD7A was produced by cDNA immunization essentially as described previously (31). An expression construct encoding full-length human THSD7A was conjugated to $1-\mu \mathrm{m}$ gold particles (Bio-Rad Laboratories). These were ballistically injected into a rabbit at the antibody core unit of the University Medical Center Hamburg-Eppendorf. The rabbit received 4 immunizations in 3- to 6-week intervals, each with 12 shots of plasmid-conjugated gold particles ( $1 \mu \mathrm{g}$ DNA/mg gold per shot). Serum was obtained 3 weeks after the last DNA immunization. The specificity of the antiserum for native human THSD7A was verified using immunofluorescence staining of Chinese hamster ovary (CHO) cells with serial dilutions of the antiserum 24 hours after transient cotransfection of cells with expression constructs for nuclear GFP and full-length THSD7A and by Western blotting with native human THSD7A from glomerular extracts, recombinant human THSD7A, and recombinant mouse THSD7A. Bound antibodies were detected with phycoerythrin-conjugated anti-rabbit IgG (Dianova). Serum K1405 was used at a dilution of 1:400 for staining of frozen kidney sections and transfected HEK293 cells (Figure 3B, Figure 6C, and Supplemental Figures 4 and 10). For FACS analyses, K1405 anti-THSD7A antibody was labeled with AF647 using the Alexa Fluor 647 antibody labeling kit according to the manufacturer's instructions and was used at a dilution of 1:400 (Supplemental Figure 11, B and C).
Electron microscopy. Electron microscopic analyses were performed on kidneys that were fixed in $4 \%$ buffered paraformaldehyde. Tissue was postfixed with $1 \%$ osmium in $0.1 \mathrm{M}$ sodium cacodylat buffer, stained with $1 \%$ uranyl acetate, and embedded in epoxy resin (Serva). Ultrathin sections were cut (Ultramicrotome; Reichert Jung) and contrasted with uranyl acetate in methanol, followed by lead citrate. Micrographs were generated with a transmission electron microscope (JEM 1010; JEOL).

Immunogold electron microscopy. Fixation for immunogold labeling: The fixation procedure and preparation of Bouin's solution for immunogold staining were modified according to Somogyi and Takagi (32). Animals were transcardially perfused with $0.1 \mathrm{M}$ phosphate buffer $(\mathrm{PB}), \mathrm{pH}$ 7.4, at $4^{\circ} \mathrm{C}$. The fixative consisted of $500 \mathrm{ml} 0.2 \mathrm{M} \mathrm{PB}, \mathrm{pH} 7.4,150 \mathrm{ml}$ saturated picric acid (Bouin's solution: $0.044 \mathrm{M}$ picric acid, $5 \%$ [v/v] acidic acid, and $10 \%$ [v/v] formaldehyde in distilled water); $346.8 \mathrm{ml}$ of $4 \%$ paraformaldehyde solution; and $3.2 \mathrm{ml}$ of $25 \%$ glutaraldehyde at a final $\mathrm{pH}$ of 7.4. The final concentrations were $4 \%$ paraformaldehyde, $0.08 \%$ glutaraldehyde, and $0.15 \%$ picric acid. Kidneys were removed after the perfusion procedure and postfixed in $100 \mathrm{ml}$ of $0.1 \mathrm{M} \mathrm{PB}$ including $4 \%$ paraformaldehyde and $0.15 \%$ picric acid for 24 hours at $4{ }^{\circ} \mathrm{C}$. Vibratome sections (Leica VT 1000S) of fixed tissue were made at $60-\mu \mathrm{m}$ thickness for areas of interest, and the slices were placed into cryoprotection solution containing $0.1 \mathrm{M} \mathrm{PB}, \mathrm{pH} 7.4,30 \%$ sucrose, $1 \%$ polyvinylpyrrolidone, and $30 \%$ ethylenglycol. Pre-embedding immunogold protocol: To remove the cryoprotection solution, the $60-\mu \mathrm{m}$ slices were washed twice in $0.1 \mathrm{M}$ sodium phosphate buffer (PBS, pH 7.4) for 20 minutes, followed by washing of the slices in 0.1 M PBS with $10 \%$ sucrose for 30 minutes and $0.1 \mathrm{M}$ PBS with 20\% sucrose for another 30 minutes. Tissue was transferred to a drop of PBS with $20 \%$ sucrose and frozen briefly with nitrogen and then thawed to RT. Subsequently, the tissue was washed 3 times in PBS, $\mathrm{pH}$ 7.4, for 10 minutes. Blocking reagent solution (BRS) was prepared with $100 \mathrm{ml}$ PBS; 800 mg BSA (SigmaAldrich; catalog A7638-5G); $250 \mu \mathrm{l} 40 \%$ cold water fish skin gelatin (AURION; CWFSG 900.033); and $162.5 \mathrm{mg}$ sodium acid. To increase the specificity of the primary antibody reaction, BRS supplemented with $2 \%$ normal goat serum (NGS) (Sigma-Aldrich) was used for 1 hour for the blocking reaction. The primary antibody was dissolved in BRS overnight at $4^{\circ} \mathrm{C}$. After 24 hours, the tissue was washed in PBS 3 times for 5 minutes each time and incubated with $12 \mathrm{~nm}$ gold-conjugated antibody solution (1:50) in BRS for 2 hours. Subsequently, the tissue was washed 3 times in PBS, pH 7.4, for 5 minutes each time, followed by $1 \%$ glutaraldehyde solution in $0.1 \mathrm{M} \mathrm{PB}$ for 10 minutes. The tissue was dissolved in $1 \% \mathrm{OsO}_{4}$ in distilled water at $4^{\circ} \mathrm{C}$ for 10 minutes, followed by dehydration in an ethanol series. Subsequently, the probes were transferred twice into propylene oxide for 5 minutes and embedded in a mixture of epon and propylene oxide (4:1) overnight. Tissue samples were cut with an ultrathin microtome (Ultracut; Reichert Jung), and thin sections were inspected in a Phillips CM 100 electron microscope. Pictures were taken using iTEM 5.2 software (Quemesa, Olympus). The primary antibodies used were: human anti-THSD7A antibody-containing or control sera (1:100) and rabbit anti-THSD7A (1:100; Atlas; catalog HPA000923) in blocking reagent solution. The following gold-conjugated secondary antibodies were used: $12 \mathrm{~nm}$ gold-AffiniPure goat antihuIgG (H+L) antibody (Jackson ImmunoResearch; catalog 109-205088) and $12 \mathrm{~nm}$ gold-AffiniPure donkey anti-rabbit $\operatorname{IgG}(\mathrm{H}+\mathrm{L})$ antibody (Jackson ImmunoResearch; catalog 711-205-152).

Immunoblotting. Protein samples were prepared for Western blot analysis by addition of $5 \times$ Laemmli buffer (1.5 M Tris-HCl, pH 6.8, 
$50 \%$ glycerol, $10 \%$ SDS, $1 \%$ bromophenol blue) and subsequent heating to $95^{\circ} \mathrm{C}$ for 9 minutes. If reducing conditions were desired, $20 \%$ $\beta$-mercaptoethanol was added to the $5 \times$ loading buffer. Proteins were separated by electrophoresis in homemade gels containing SDS and $7 \%$ acrylamide in an electrophoresis chamber (Bio-Rad) in the presence of a migration buffer (25 mM Tris, $192 \mathrm{mM}$ glycine, 0.1\% SDS; Amresco). Proteins were then transferred to methanol-soaked PVDF membranes (EMD Millipore) under semi-dry conditions in the presence of $25 \mathrm{mM}$ Tris, $\mathrm{pH}$ 8.5, $192 \mathrm{mM}$ glycine, 20\% ethanol using Transblot Turbo (Bio-Rad) at $25 \mathrm{~V}$ constant voltage for 35 minutes. Membranes were blocked overnight at $4^{\circ} \mathrm{C}$ in $5 \%$ dry milk with PBSTween $0.05 \%$ (PBS-T) and then incubated with primary and secondary antibodies for 2 hours at RT. Primary antibodies were diluted with $0.5 \%$ dry milk in PBS-T and HRP-conjugated secondary antibodies in PBS-T alone. Membranes were washed 3 times for 5 minutes in PBS-T after incubation with primary and secondary antibodies.

If serum was used as the primary antibody, a dilution of 1:100 was used. For specific detection of THSD7A, we used a rabbit polyclonal antibody (1:1,000; Atlas). The secondary antibodies used were: HRP-conjugated goat anti-mouse IgG, goat anti-huIgG, and goat anti-rabbit IgG (all 1:20,000 and all from SouthernBiotech; catalogs 1010-05, 2040-05, and 4010-05, respectively).

Immunoprecipitation. For the isolation of glomeruli from BALB/c mice, mice were perfused with Dynabeads (Dynal) as described previously (33). MGEs $(50 \mu \mathrm{g})$ were mixed with 5 times the volume of $20 \mathrm{mM}$ Tris, $\mathrm{pH} 7.4,140 \mathrm{mM} \mathrm{NaCl}, 1 \mathrm{mM} \mathrm{CaCl}_{2}$, and $5 \mu \mathrm{l}$ serum from a patient with THSD7A-associated MN or from a healthy donor. Samples were incubated 2 hours at $4^{\circ} \mathrm{C}$ on a rotator. Next, $20 \mu$ Protein G Affinity Matrix (GeneScript) was added, and samples were incubated overnight at $4^{\circ} \mathrm{C}$ on a rotator. Samples were then centrifuged for 1 minute at low speed, and the supernatant was discarded. The pellet was washed 3 times with $20 \mathrm{mM}$ Tris, $\mathrm{pH} 7.4,140 \mathrm{mM} \mathrm{NaCl}, 0.8 \mathrm{mM} \mathrm{CaCl}_{2}, 0.02 \% \mathrm{SDS}, 0.2 \%$ $\mathrm{NP}-40$, and $0.1 \% \mathrm{Na}-\mathrm{DOC}$ and subsequently resuspended in Laemmli buffer containing $\beta$-mercaptoethanol, loaded on polyacrylamide gels, and electrophoresed. Immunoprecipitates were detected with the rabbit polyclonal anti-THSD7A antibody (Atlas), as described above.

Antibody elution. Sixty to seventy $20-\mu \mathrm{m}$ sections from frozen renal tissue were resuspended in $1 \mathrm{ml}$ sterile PBS and centrifuged at low speed for 1 minute. The pellet was then washed 3 times in $1 \mathrm{ml} \mathrm{PBS}$. After the last wash, the pellet was resuspended in $150 \mu \mathrm{l}$ of $25 \mathrm{mM}$ citrate, $\mathrm{pH}$ 3.2, and left on ice for 20 minutes, with intermediate shaking. Subsequently, the sample was centrifuged again, and the supernatant was added to $150 \mu \mathrm{l}$ of $1 \mathrm{M}$ Tris, $\mathrm{pH}$ 8. The pellet was resuspended in $150 \mu \mathrm{l}$ of $25 \mathrm{mM}$ citrate, $\mathrm{pH} 2.5$, and left on ice for 20 minutes, with intermediate shaking. The sample was then centrifuged for 5 minutes at $20,817 \mathrm{~g}$ at $4^{\circ} \mathrm{C}$. Next, the supernatant was added to the first elution and diluted to $2 \mathrm{ml}$ using $0.05 \%$ dry milk in PBS-T. The resulting sample was used as the primary antibody in immunoblot analyses, as described above.

Albumin ELISA. Urine albumin content was quantified using a commercially available ELISA system (Bethyl) according to the manufacturer's instructions. Briefly, 96-well plates were coated 1:100 with goat anti-mouse albumin in binding buffer $(0.05 \mathrm{M}$ carbonate-bicarbonate, $\mathrm{pH}$ 9.6) for 60 minutes at RT. After washes in $50 \mathrm{mM}$ Tris, $0.14 \mathrm{M} \mathrm{NaCl}$, and $0.05 \%$ Tween-20, pH 8.0, the plates were blocked for 30 minutes at RT with $50 \mathrm{mM}$ Tris, $0.14 \mathrm{M} \mathrm{NaCl}$, and 1\% BSA, pH 8.0, and rewashed. Diluted urine was incubated for 60 minutes at RT. Following the washes, the secondary antibody (1:40,000; HRP goat anti-mouse albumin) was applied for 60 minutes at RT. After the washes, enzyme substrate included in the kit was added, and the color development was stopped after 5 minutes with $2 \mathrm{M}$ sulphuric acid. Extinction was measured at 450 $\mathrm{nm}$ in an ELISA plate reader (BioTek; ELx808). The urinary albumin concentration was calculated according to the formula for absorption: $(\mathrm{A}-\mathrm{D}) / 1+(x / \mathrm{C})^{\mathrm{B}}+\mathrm{D}$, where $\mathrm{A}$ and $\mathrm{D}$ are values from the standard curve. Regression values for the standard curve were calculated to assess the accuracy of the measured values. Standard curves with $r$ values greater than 0.9950 were used. The urinary albumin values were standardized against urinary creatinine values of the same sample.

Purification of human THSD7A and anti-THSD7A antibodies. Human DDK-tagged THSD7A (huTHSD7A) was expressed in HEK293 cells as described above. Forty-eight hours after transfection, cells were scraped in $50 \mathrm{mM}$ Tris, pH 7.4, $150 \mathrm{mM} \mathrm{NaCl}, 1 \mathrm{mM}$ EDTA, 1\% Triton $\mathrm{X}-100$, and protease inhibitor cocktail. Cells were sonicated shortly, incubated 1 hour at $4^{\circ} \mathrm{C}$ with slow rotation, and then centrifuged for 10 minutes at 20,000 $\mathrm{g}$. A 2-ml chromatographic column was rinsed several times with PBS before addition of $1 \mathrm{ml}$ anti-Flag M2 affinity gel (Sigma-Aldrich). The resin was equilibrated with a total of $15 \mathrm{ml}$ PBS containing $0.2 \mathrm{mg} / \mathrm{ml} \mathrm{HSA}$ and $1 \%$ Triton X-100. Subsequently, the cell lysate containing DDK-tagged huTHSD7A was loaded on the column. The flow-through was collected and loaded again. The column was then washed with $10 \mathrm{ml}$ PBS containing $0.2 \mathrm{mg} / \mathrm{ml} \mathrm{HSA}$ and $1 \%$ Triton X-100. Next, bound huTHSD7A was eluted in 3 steps with 2.25 $\mathrm{ml}$ each of $100 \mathrm{mM}$ glycine, $\mathrm{pH} 3.5$, containing $0.2 \mathrm{mg} / \mathrm{ml} \mathrm{HSA}$ and $1 \%$ Triton X-100. In order to change the buffer, eluates were loaded onto PD-10 columns (GE Healthcare), the flow-through was discarded, and the columns were rinsed with $3 \mathrm{ml}$ PBS containing $0.2 \mathrm{mg} / \mathrm{ml} \mathrm{HSA}$ and $1 \%$ Triton $\mathrm{X}-100$. Purification was verified using Western blot analysis, and purified THSD7A was quantified by Coomassie blue staining. Since approximately $95 \%$ of huTHSD7A was in the first 2 eluates, the eluates were pooled and concentrated using Amicon Ultra-15 centrifugal filters with a molecular cut-off of $100 \mathrm{kDa}$ (EMD Millipore).

For purification of anti-THSD7A antibodies from serum from a patient with THSD7A-associated MN, we coupled purified huTHSD7A protein to beaded agarose using the AminoLink Plus Immobilization Kit (Thermo Scientific) according to the manufacturer's instructions, with some modifications. Briefly, $250 \mu$ purified huTHSD7A was mixed with $1 \mathrm{ml}$ coupling buffer and loaded on a chromatography column. After addition of $20 \mu \mathrm{l}$ sodium cyanoborohydride solution, the column was stored overnight at $4^{\circ} \mathrm{C}$ with gentle agitation. Subsequently, the column was washed twice with $1 \mathrm{ml}$ quenching buffer, 4 times with $1 \mathrm{ml}$ wash solution, and 3 times with $1 \mathrm{ml}$ storage buffer. The column was then rinsed twice with PBS, and $10 \mathrm{ml}$ serum containing anti-THSD7A antibodies was loaded onto the column. The flow-through was taken and loaded again. The column was then washed with $10 \mathrm{ml}$ PBS containing $2 \%$ mouse serum. Elution was performed 2 times with $2.25 \mathrm{ml}$ of $100 \mathrm{mM}$ glycine, $\mathrm{pH} 3.5$. Subsequently, the column was washed again with $10 \mathrm{ml}$ PBS containing $2 \%$ mouse serum, and the flow-through from the first round was loaded again 2 times with subsequent elution. This procedure was repeated 3 times, resulting in 1 final flow-through devoid of anti-THSD7A antibodies (herein referred to as depleted serum) and a total of 6 eluates (herein referred to as E1-E6) containing the pulled anti-THSD7A antibodies. The eluate buffer was changed to PBS only using PD-10 columns as described above. Aliquots were taken at all steps of the experiment for Coomassie blue staining and Western blot analysis. Finally, eluates 
were pooled and concentrated using Amicon Ultra-15 centrifugal filters with a molecular cut-off of $100 \mathrm{kDa}$ (EMD Millipore). The reactivity of purified anti-THSD7A antibodies with THSD7A was tested using different dilutions in the Western blot analysis, compared with whole serum reactivity, and adjusted to equal amounts.

Cell culture, cell transfection, and in vitro experiments. HEK293 cells were cultured in DMEM supplemented with $10 \% \mathrm{FCS}, 100 \mathrm{U} / \mathrm{ml}$ penicillin, and $100 \mathrm{mg} / \mathrm{ml}$ streptomycin (all from Invitrogen) at $37^{\circ} \mathrm{C}$ and $5 \% \mathrm{CO}_{2}$. cDNAs coding for human and murine THSD7A cloned into the pCMV6 entry vector containing a DDK tag were purchased from OriGene. HEK293 cells were transiently transfected using the calcium phosphate method. For each $10-\mathrm{cm}$ dish of cultured cells, we used 15 $\mu \mathrm{g}$ plasmid DNA mixed with $36 \mu \mathrm{l}$ of $2 \mathrm{M} \mathrm{CaCl}_{2}$ and diluted with sterile water up to a volume of $300 \mu$ l. This solution was gently mixed with an equal volume of $2 \times$ HEPES-buffered saline (HBS) $(275 \mathrm{mM} \mathrm{NaCl}, 55$ mM HEPES, pH 7.0) and incubated for 30 minutes at RT. The resulting solution was added drop-wise to the cells. The medium was changed to serum-free medium 24 hours after transfection. Forty-eight hours after transfection, cells were treated as described below.

For quantification and FACS analyses of detached cells, cell culture medium was collected 60 minutes after treatment and centrifuged for 5 minutes at $100 \mathrm{~g}$. The cell pellet was resuspended in PBS and centrifuged again. For quantification of detached cells, cell pellets were then lysed in $50 \mathrm{mM}$ Tris, pH 7.4, $150 \mathrm{mM} \mathrm{NaCl}, 0.1 \%$ SDS, $1 \%$ NP-40, and $0.5 \% \mathrm{Na}-\mathrm{DOC}$ supplemented with protease inhibitor cocktail as described above. Protein concentration was measured using the Pierce BCA protein assay kit according to the manufacturer's instructions. Cell detachment experiments were conducted 10 times, each time with $2 \%$ of both anti-THSD7A antibody-positive sera, MN 1 and MN 2, and serum from a healthy donor. Experiments were also performed with 5 different sera from patients with anti-PLA ${ }_{2} \mathrm{R} 1$ antibody-positive $\mathrm{MN}$ and 5 different sera from patients with anti-THSD7A and anti$\mathrm{PLA}_{2} \mathrm{R} 1$ antibody-negative MN. Additionally, experiments were performed 5 times with affinity-purified anti-THSD7A antibodies, serum depleted of anti-THSD7A antibodies (2\%), and affinity-purified IgG from a healthy donor. The amount of purified anti-THSD7A antibody was adapted to the anti-THSD7A antibody-containing whole serum by analysis of reactivity in Western blot analysis. For FACS analyses, cells were resuspended in MACS buffer (Miltenyi Biotec) and stained with AF647 anti-THSD7A (1:400) and with Cy2 anti-huIgG (1:200) for 20 minutes at $4^{\circ} \mathrm{C}$. For the detection of cell death, the LIVE/DEAD kit (Thermo Fisher Scientific) was used according to the manufacturer's instructions. Experiments were performed using a BD LSR II Flow Cytometer and analyzed with FlowJo software. FACS experiments were conducted 3 independent times with MN serum 1 .

Phase-contrast micrographs were taken with an Axiovert 40 microscope using Axiovision software (both from Zeiss). For immunofluorescence analyses, HEK293 cells were fixed with ice-cold 100\% methanol for 10 minutes at $-20^{\circ} \mathrm{C}$ or with $4 \%$ paraformaldehyde for 8 minutes at RT. Staining was performed as described for the frozen tissue sections, using Cy2 anti-huIgG (1:200; Dianova) and K1405 anti-THSD7A antibody (1:400) for the visualization of THSD7A expression and autoantibody binding to HEK293 cells, and phalloidin-AF568 (1:200; Molecular Probes; catalog A12380) for visualization of cytoskeletal rearrangement.

GEC culture. For the isolation of glomeruli, mice were perfused with Dynabeads (Dynal) as described previously $(33,34)$. Decapsulated glomeruli were plated on collagen 1 -coated 10 -cm culture plates
(Biocoat) in RPMI 1640 supplemented with 10\% FCS, 10 mmol/1 HEPES, $1 \mathrm{mmol} / 1$ sodium pyruvate, $100 \mathrm{U} / \mathrm{ml}$ penicillin, and $100 \mathrm{mg} /$ $\mathrm{ml}$ streptomycin at $38^{\circ} \mathrm{C}$ and $5 \% \mathrm{CO}_{2}$. Three to five days after the initial glomerular isolation, primary culture GECs were challenged with 1:50 (2\%) anti-THSD7A antibody-containing patient's serum or serum from a healthy donor. Experiments were conducted independently 3 times with 2 anti-THSD7A antibody-containing sera (MN 1 and MN 2) and 2 different control sera. Moreover, cells were treated with affinity-purified anti-THSD7A antibodies, serum depleted of antiTHSD7A antibodies, and affinity-purified IgG from a healthy donor. These experiments were conducted independently 2 times (with 10 for each condition). Immunofluorescence analyses were performed 40-60 minutes after serum challenge. For immunofluorescence analyses, GECs were fixed with $4 \%$ paraformaldehyde for 8 minutes at RT. Staining was performed as described for the frozen tissue sections. The following primary antibodies were used: Cy2-coupled anti-huIgG (1:100; Dianova); $\alpha$-actinin-4 (1:200; ImmunoGlobe; catalog 004205); p-paxillin (Tyr118) (1:400; Thermo Fischer Scientific; catalog 44-722G); and phalloidin-AF568 (1:200; Molecular Probes).

F-actin quantification was performed using ImageJ software (NIH). For analysis, grayscale images from 3 independent experiments (with at least 4 per condition) were used at a 1,000-fold magnification. Individual cell areas were circled, and optical density was analyzed.

Statistics. Data are expressed as the mean \pm SEM. For statistical analysis for comparison of mouse treatment groups, we performed a 2-tailed, nonparametric Mann-Whitney $U$ test to enable robust conclusions on effect significance in the event of departures from normality associated with small sample sizes. The number of animals used for the experiments was limited by the availability of serum from patients with THSD7A-associated MN. Therefore, no statistical method was used to predetermine sample size. Replicates used were biological replicates, which were measured using different samples derived from distinct mice. All animals were WT mice and were blindly assigned to the experimental groups. No inclusion or exclusion criteria were defined for animals, and no animals were excluded from the analysis. Examiners were not blinded, except for those conducting the following in vitro analyses: serum treatment of GECs and transfected HEK293 cells for all experiments, phase-contrast microscopy of nondetached GECs and HEK293 cells after treatment, culture medium withdrawal for protein quantification of detached HEK293 cells and FACS analyses, protein quantification of detached cells, sample preparation for Western blot analyses, and all histological stainings. A $P$ value of less than 0.05 was considered significant.

Study approval. All patients provided written, informed consent, and the study was approved by the local ethics committee and conducted according to Declaration of Helsinki guidelines. All animal experiments were performed according to national and institutional animal care and ethics guidelines and were approved by the veterinary agency and the local animal care committee of Hamburg, Germany (BSG Amt für Gesundheit und Verbraucherschutz, Lebensmittelsicherheit und Veterinärwesen).

\section{Author contributions}

NMT conducted animal experiments, performed protein and antibody purification and in vitro studies, analyzed the data, and wrote the first draft of the manuscript. EH acquired and analyzed patients' clinical data and measured autoantibody titers. ATR per- 
formed FACS analyses. LF and GR performed immunogold electron microscopic studies. UH performed histologic analyses of human biopsy samples and conventional electron microscopy. JG, $\mathrm{FB}$, and $\mathrm{KB}$ provided reagents and acquired clinical data. GZ provided reagents. FKN generated a rabbit antibody for the detection of THSD7A. GL designed some of the protein biochemical experiments. CMS designed and conducted animal experiments, generated GECs, performed confocal microscopy, and revised the manuscript. RAKS designed the study and finalized the manuscript.

\section{Acknowledgments}

These studies were supported by grants from the Deutsche Forschungsgemeinschaft, KFO 228 (STA-193/9-2) and the Sonderforschungsbereich 1192 (projects B1, B2, and B3). We would like to thank Wolfgang Schlumberger of EUROIMMUN AG (Lübeck,
Germany) for providing the indirect immunofluorescence test (IFT), which allowed the measurement of THSD7A serum antibody titers in patients. We thank Ursula Kneißler and Barbara Holstermann for performing the electron microscopic studies. We thank Eugen Kinzler, Samaneh Liagos, and Daniela Bergleiter for following the patients and collecting sera. We also thank Marlies Sachs, Marion Nissen, Silke Dehde, and Olga Ilin for their excellent technical assistance.

Address correspondence to: Catherine Meyer-Schwesinger or Rolf A.K. Stahl, III. Medizinische Klinik, Universitätsklinikum Hamburg-Eppendorf, Martinistrasse 52, D-20246 Hamburg, Germany. Phone: 49.152.22815839; E-mail: c.meyer-schwesinger@ uke.de (C. Meyer-Schwesinger). Phone: 49.152.22816815; E-mail: rstahl@uke.de (R.A.K.Stahl).
1. Heymann W, Hackel DB, Harwood S, Wilson SG, Hunter JL. Production of nephrotic syndrome in rats by Freund's adjuvants and rat kidney suspensions. Proc Soc Exp Biol Med. 1959;100(4):660-664.

2. Kerjaschki D, Neale TJ. Molecular mechanisms of glomerular injury in rat experimental membranous nephropathy (Heymann nephritis). JAm Soc Nephrol. 1996;7(12):2518-2526.

3. Farquhar MG, Saito A, Kerjaschki D, Orlando RA. The Heymann nephritis antigenic complex: megalin (gp330) and RAP. JAm Soc Nephrol. 1995;6(1):35-47.

4. Kerjaschki D, Farquhar MG. The pathogenic antigen of Heymann nephritis is a membrane glycoprotein of the renal proximal tubule brush border. Proc Natl Acad Sci USA . 1982;79(18):5557-5561.

5. Salant DJ, Belok S, Madaio MP, Couser WG. A new role for complement in experimental membranous nephropathy in rats. J Clin Invest. 1980;66(6):1339-1350.

6. Debiec H, et al. Antenatal membranous glomerulonephritis due to anti-neutral endopeptidase antibodies. N Engl JMed. 2002;346(26):2053-2060.

7. Beck LH Jr. M-type phospholipase A2 receptor as target antigen in idiopathic membranous nephropathy. N Engl J Med. 2009;361(1):11-21.

8. Tomas NM, et al. Thrombospondin type-1 domaincontaining 7A in idiopathic membranous nephropathy. N Engl JMed. 2014;371(24):2277-2287.

9. Vivarelli M, et al. Genetic homogeneity but IgG subclass-dependent clinical variability of alloimmune membranous nephropathy with antineutral endopeptidase antibodies. Kidney Int. 2015;87(3):602-609.

10. Stahl R, Hoxha E, Fechner K. PLA2R autoantibodies and recurrent membranous nephropathy after transplantation. $N$ Engl J Med. 2010;363(5):496-498.

11. Seitz-Polski B, et al. Prediction of membranous nephropathy recurrence after transplantation by monitoring of anti-PLA2R1 (M-type phospholipase A2 receptor) autoantibodies: a case series of 15 patients. Nephrol Dial Transplant. 2014;29(12):2334-2342.

12. Hoxha E, Harendza S, Pinnschmidt H, Panzer U, Stahl RA. PLA2R antibody levels and clinical out- come in patients with membranous nephropathy and non-nephrotic range proteinuria under treatment with inhibitors of the renin-angiotensin system. PLoS One. 2014;9(10):e110681.

13. Hoxha E, Thiele I, Zahner G, Panzer U, Harendza S, Stahl RA. Phospholipase A2 receptor autoantibodies and clinical outcome in patients with primary membranous nephropathy. JAm Soc Nephrol. 2014;25(6):1357-1366.

14. Hoxha E, Harendza S, Pinnschmidt H, Panzer U, Stahl RA. M-type phospholipase A2 receptor autoantibodies and renal function in patients with primary membranous nephropathy. Clin J Am Soc Nephrol. 2014;9(11):1883-1890.

15. Kanigicherla D, et al. Anti-PLA2R antibodies measured by ELISA predict long-term outcome in a prevalent population of patients with idiopathic membranous nephropathy. Kidney Int . 2013;83(5):940-948.

16. Meyer-Schwesinger C, Lambeau G, Stahl RA. Thrombospondin type-1 domain-containing 7A in idiopathic membranous nephropathy. N EnglJ Med. 2015;372(11):1074-1075.

17. Gödel M, Grahammer F, Huber TB. Thrombospondin type-1 domain-containing 7A in idiopathic membranous nephropathy. $N$ Engl J Med. 2015;372(11):1073.

18. Prunotto M, et al. Autoimmunity in membranous nephropathy targets aldose reductase and SOD2. J Am Soc Nephrol. 2010;21(3):507-519.

19. Kerjaschki D, et al. Transcellular transport and membrane insertion of the C5b-9 membrane attack complex of complement by glomerular epithelial cells in experimental membranous nephropathy. J Immunol. 1989;143(2):546-552.

20. Zamir E, Geiger B. Molecular complexity and dynamics of cell-matrix adhesions. J Cell Sci. 2001;114(pt 20):3583-3590.

21. Turner CE, Glenney JR, Burridge K. Paxillin: a new vinculin-binding protein present in focal adhesions. J Cell Biol. 1990;111(3):1059-1068.

22. Burridge K, Turner CE, Romer LH. Tyrosine phosphorylation of paxillin and pp $125 \mathrm{FAK}$ accompanies cell adhesion to extracellular matrix: a role in cytoskeletal assembly. JCell Biol. 1992;119(4):893-903.

23. Beck LH Jr, Salant DJ. Membranous neph- ropathy: from models to man. J Clin Invest. 2014;124(6):2307-2314.

24. Baker PJ, Ochi RF, Schulze M, Johnson RJ, Campbell C, Couser WG. Depletion of C6 prevents development of proteinuria in experimental membranous nephropathy in rats. Am J Pathol. 1989;135(1):185-194.

25. Spicer ST, et al. Induction of passive Heymann nephritis in complement component 6-deficient PVG rats. JImmunol. 2007;179(1):172-178.

26. Leenaerts PL, Hall BM, Van Damme BJ, Daha MR, Vanrenterghem YF. Active Heymann nephritis in complement component C6 deficient rats. Kidney Int. 1995;47(6):1604-1614.

27. Kerjaschki D, et al. Pathogenic antibodies inhibit the binding of apolipoproteins to megalin/gp330 in passive Heymann nephritis. J Clin Invest. 1997;100(9):2303-2309.

28. Buelli S, et al. Mitochondrial-dependent autoimmunity in membranous nephropathy of IgG4related disease. EBioMedicine. 2015;2(5):456-466.

29. Dähnrich C, et al. Development of a standardized ELISA for the determination of autoantibodies against human M-type phospholipase A2 receptor in primary membranous nephropathy. Clin Chim Acta. 2013;421:213-218.

30. Hoxha E, et al. An immunofluorescence test for phospholipase- $\mathrm{A}_{2}$-receptor antibodies and its clinical usefulness in patients with membranous glomerulonephritis. Nephrol Dial Transplant. 2011;26(8):2526-2532.

31. Möller S, et al. Monitoring the expression of purinoceptors and nucleotide-metabolizing ecto-enzymes with antibodies directed against proteins in native conformation. Purinergic Signal. 2007;3(4):359-366.

32. Somogyi P, Takagi H. A note on the use of picric acid-paraformaldehyde-glutaraldehyde fixative for correlated light and electron microscopic immunocytochemistry. Neuroscience. 1982;7(7):1779-1783.

33. Takemoto M, et al. A new method for large scale isolation of kidney glomeruli from mice. Am J Pathol. 2002;161(3):799-805.

34. Liu X, et al. Isolating glomeruli from mice: A practical approach for beginners. Exp Ther Med. 2013;5(5):1322-1326. 